\title{
WestVirginiaUniversity
}

THE RESEARCH REPOSITORY @ WVU

Graduate Theses, Dissertations, and Problem Reports

2012

\section{The Relationship between Temperament and Facets of Creativity}

Clark Daniel Pinson

West Virginia University

Follow this and additional works at: https://researchrepository.wvu.edu/etd

\section{Recommended Citation}

Pinson, Clark Daniel, "The Relationship between Temperament and Facets of Creativity" (2012). Graduate Theses, Dissertations, and Problem Reports. 3493.

https://researchrepository.wvu.edu/etd/3493

This Dissertation is protected by copyright and/or related rights. It has been brought to you by the The Research Repository @ WVU with permission from the rights-holder(s). You are free to use this Dissertation in any way that is permitted by the copyright and related rights legislation that applies to your use. For other uses you must obtain permission from the rights-holder(s) directly, unless additional rights are indicated by a Creative Commons license in the record and/ or on the work itself. This Dissertation has been accepted for inclusion in WVU Graduate Theses, Dissertations, and Problem Reports collection by an authorized administrator of The Research Repository @ WVU.

For more information, please contact researchrepository@mail.wvu.edu. 
The Relationship between Temperament and Facets of Creativity

\title{
Clark Daniel Pinson
}

\author{
Dissertation submitted to the \\ College of Human Resources and Education \\ at West Virginia University \\ in partial fulfillment of the requirements \\ for the degree of
}

\author{
Doctor of Philosophy \\ in \\ Counseling Psychology
}

Jeffrey A. Daniels, Ph.D., Chair

Tandy J. McClung, Ed.D.

Helen A. Panzironi, BFA, MSW

Christine J. Schimmel, Ed.D.

Roy H. Tunick, Ed.D.

Richard T. Walls, Ph.D.

Department of Counseling, Rehabilitation Counseling, and Counseling Psychology

\author{
Morgantown, West Virginia \\ 2012
}

Keywords: creativity, psychobiological model of temperament and character, bipolar disorders

Copyright 2012 Clark Daniel Pinson 


\section{ABSTRACT \\ The Relationship between Temperament and Facets of Creativity Clark Daniel Pinson}

The purported association between mental illness and creativity has been most heavily researched in individuals diagnosed with a bipolar disorder. The bipolar disorders have been conceptualized as a spectrum of disorders that range from problematic and labile affect to different types of temperament. Temperament has classically been defined as elements of personality that are heritable, stable, based on emotion, and uninfluenced by socio-cultural learning. Contemporary literature suggested that at least some aspects of creativity may be enhanced by an affective range that has been best characterized by the milder part of the bipolar spectrum of disorders. The primary inquiry of this study was to investigate whether the creative advantages linked to the milder part of the bipolar continuum may be due to a specific type (or types) of temperament. The psychobiological model of temperament and character was utilized to provide a theoretical context for which to consider the possible relationship between traits and aspects of creativity. A multivariate analysis of covariance (MANCOVA) was non-significant such that creativity groups could not be differentiated by any dimensions of temperament and character. Limitations of the current study were reviewed and recommendations for future research were offered. 


\section{Acknowledgements}

I could not have finished this project without the love and support of my parents. Their ongoing encouragement was essential to the genesis of this document. For example, they graciously provided me with financial support during three months when I did not maintain a job in order to completely focus on writing the majority of chapter two. In fact, I do not think I would have been able to endure the rigors and stressors of graduate school without them.

I would never have refined the ideas for this project nor would I have been willing to pursue the actual research without the guidance of Helen Panzironi, BFA, MSW and the people of the West Virginia Center for Excellence in Disabilities (WVU CED). A research stipend provided by the WVU CED, which was managed by Helen, was the impetus for my initial exploration into the putative association between bipolarity and creativity. Furthermore, the ideas contained within this document were influenced by my many discussions about the topic with Helen. She also helped facilitate a meeting where I was able to share my ideas with William Winsor of the WVU College of Creative Arts (WVU CAC) and secure his assistance in ascertaining participants for this study.

I also want to thank the other members of my dissertation committee. Dr. Jeffrey Daniels, Ph.D. was an excellent dissertation chair and I valued his prompt and insightful feedback throughout this process. I am grateful for the statistical consultations and proofreading provided by Dr. Richard Walls, Ph.D. I appreciate the additional contributions provided by Dr. Tandy McClung, Ed.D., Dr. Christine Schimmel, Ed.D., and Dr. Roy Tunick, Ed.D. Finally, I want to thank former committee members Dr. Jennifer Adams, Ph.D. and Dr. Melanie Nuszkowski, Ph.D. for their efforts during my proposal. 


\section{Table of Contents}

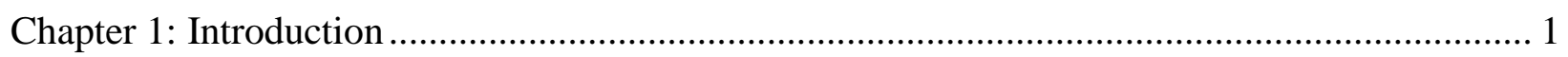

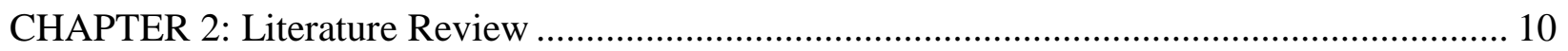

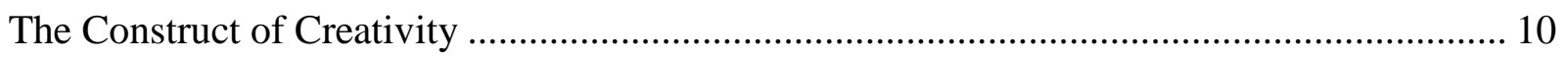

The psychobiological model of temperament and character ............................................... 20

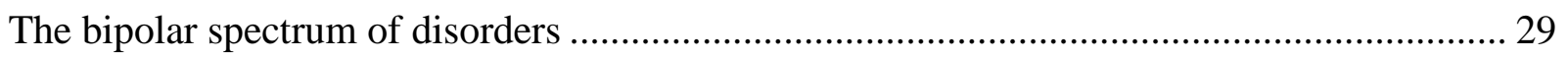

Research on the Association between Bipolarity and Creativity ......................................... 38

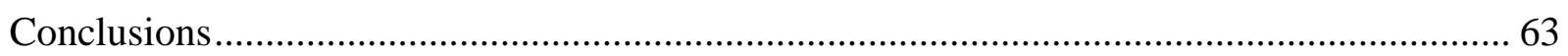

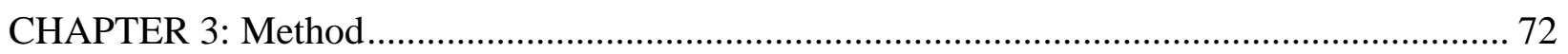

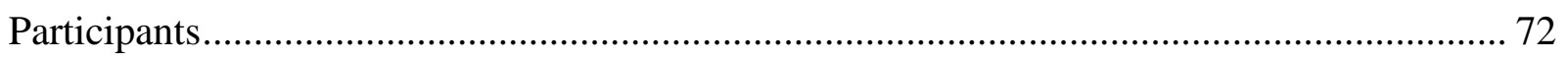

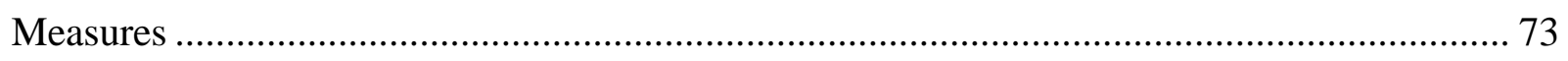

The Barron-Welsh Art Scale (BWAS) ..................................................................... 73

The Temperament and Character Inventory - Revised (TCI-R) .................................... 74

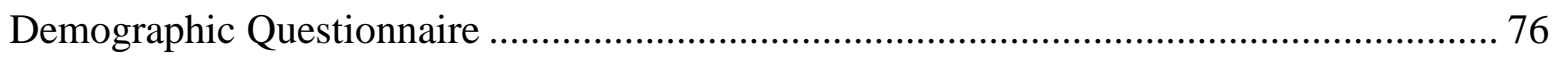

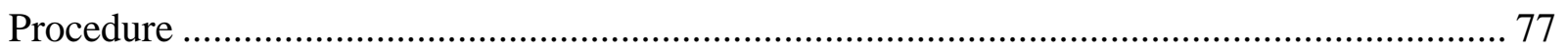

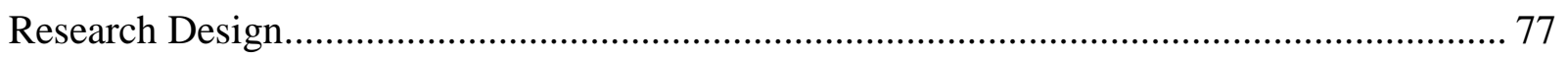

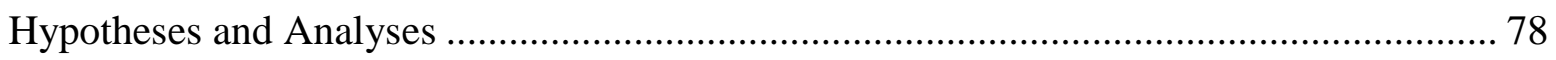

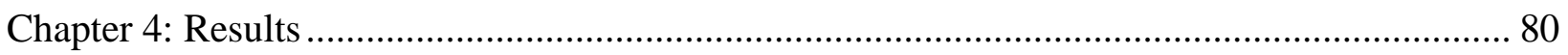

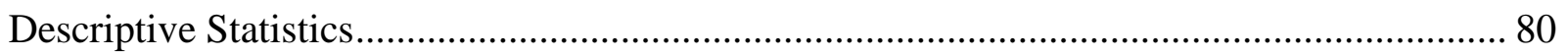

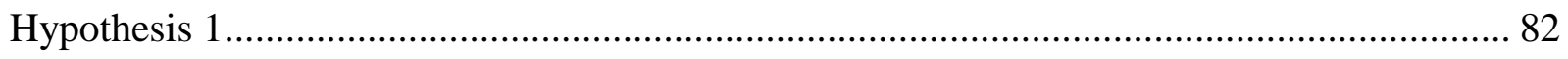

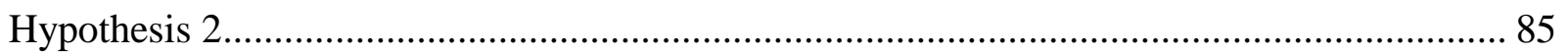

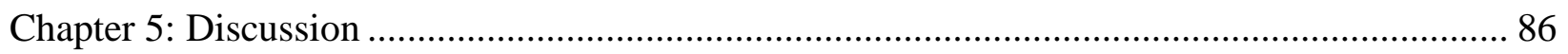

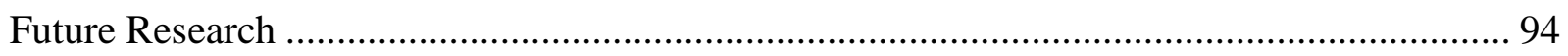

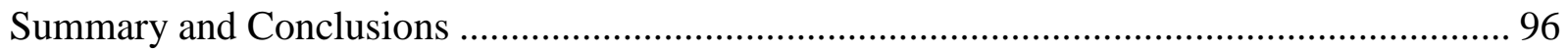

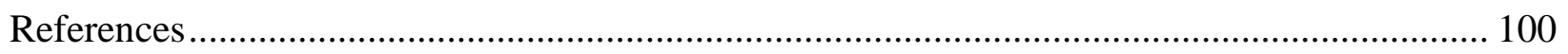

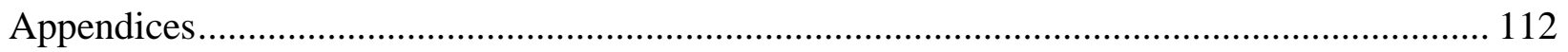




\section{List of Tables}

Table 1 Frequencies and Percentages for Participant Demographics ..................................... 80

Table 2 Means and Standard Deviations for Temperament and Character Percentile Scores...... 82

Table 3 Means and Standard Deviations for Temperament and Character Percentile Scores...... 84

Table 4 Means and Standard Deviations for Self-Directedness, Cooperativeness, Self-

Transcendence, Reward Dependence, Harm Avoidance, and Novelty Seeking by Creativity

Group 85 


\section{Chapter 1: Introduction}

Many people value creativity. The creative process is associated with the genesis of art, literature, music, dance, cinema, invention, and scientific discoveries (Rothenberg, 2001). The contribution of creativity has also been linked to workplace leadership, vocational and life success, psychological coping and emotional growth, maintenance of relationships, and effective therapeutic treatments (Makel \& Plucker, 2008; Plucker, Beghetto, \& Dow, 2004). Two major journals are dedicated to creativity research and theory: Creativity Research Journal and the Journal of Creative Behavior, and several more regularly include articles about creativity (e.g., Empirical Studies of the Arts, Imagination, Cognition, and Personality) (Baer \& Kaufman, 2006; Simonton, 2002).

There is not a standard operational definition of creativity in the research literature (Andreasen, 2008; Benham, 2005; Feldhusen \& Goh, 1995; Makel \& Plucker, 2008; Mumford, 2003; Plucker et al., 2004; Prentky, 2001; Smith, 2005; Sternberg, 2006; Waddell, 1998). Disagreement regarding most aspects of creativity persists. It is a construct that incorporates many possible elements that render it difficult to identify and define (Baer \& Feldhusen \& Goh, 1995; Kaufman, 2006; Sternberg, 2006). Whereas early authors attempted to designate one simple and universal mechanism to account for creativity (e.g., blind variation, divergent thinking, motivation, etc.), it is now commonly proposed that the construct is a complex phenomenon that consists of myriad influences (Feldhusen \& Goh, 1995; Mumford, 2003; Simonton, 2002).

Creativity is difficult to define for three additional reasons. First, it is neither entirely domain specific nor domain general (Feldhusen \& Goh, 1995; Mumford, 2003; Simonton, 2002; Sternberg, 2006). Second, there is dissent about whether everyday and eminent creativity are 
qualitatively or quantitatively distinct (Baer \& Kaufman, 2006; Feldhusen \& Goh, 1995;

Richards, 2001; Simonton, 2002). Third, it has not been studied enough (Sternberg, 2006).

Many individuals throughout history have speculated about a possible relationship between creativity and mental illness. Aristotle is often cited as having theorized that individuals who achieved eminence in philosophy, politics, poetry, and the arts had depressive tendencies (Becker, 2001; Prentky, 2001; Simonton, 1999; Waddell, 1998). However, Becker (2001) argued that Aristotle employed "melancholia" to describe a type of individual who, depending on his particular balance of "humors," could be either a successful (and sane) individual or a "madman."

The term genius was first utilized in reference to creative individuals in the $18^{\text {th }}$ century (Andreasen, 2008; Becker, 2001; Waddell, 1998). During this time period, individuals who were characterized as creative geniuses were thought to possess judgment and reason that served to counter their eccentricity, sensitivity, moodiness, and solitary lifestyle. There is no record of a purported link between genius and insanity at that time. However, the connotation of the term began to shift in the late $18^{\text {th }}$ and early $19^{\text {th }}$ centuries due to the decreasing social status of artists. In his description of this changing zeitgeist, Becker (2001) stated that "although the idea of genius may have commanded a degree of respect, even reverence, the men of genius themselves, faced with a practical world, lacked the special sense of identity necessary to separate themselves effectively from the masses" (p. 48).

Becker (2001) identified the romantic artists as the people who began to equate the creative genius with symptoms of "divine madness.” Therefore, “divine madness” became the necessary distinction of extraordinary individuals such that notions of possession and suffering were integrated into what it meant to be a creative genius. Becker suggested this association soon 
became prevalent in the self-descriptions of artists and were noted by early psychiatrists in the absence of empirical scrutiny of the proposed link. In summary, "although the Enlightenment tended to reward creative individuals who were healthy and rational with the distinction of genius, the $19^{\text {th }}$ and $20^{\text {th }}$ centuries have shown a distinct preference for those creative individuals who are diseased” (Becker, 2001, p. 52).

Becker (2001) indicated he was not suggesting that the possible relationship between creativity and mental illness was entirely the result of strict adherence to specific role expectations by artists. Instead, he was emphasizing that the history of social definitions should not be ignored in the ongoing exploration of this possible association. In fact, many researchers have identified a need for more empirical investigation of the subject. Some maintain that it is likely that scientific inquiry will eventually establish a relationship between creativity and mental health rather than mental illness (Andreasen, 2008; Prentky, 2001; Richards, 2001; Rothenberg, 2001; Waddell, 1998). I believe a person's creativity can be associated with positive aspects of her mental health regardless of whether she manifests symptoms of psychological pathology. This is consistent with one of the five unifying themes of counseling psychology (i.e., emphasis on a person's assets and strengths) as identified by Gelso and Fretz (2001).

The purported relationship between mental illness and creativity has been most heavily researched in individuals diagnosed with affective disorders (particularly the bipolar disorders) (Akiskal \& Akiskal, 1988; Akiskal, Savino, \& Akiskal, 2005; Andreasen \& Glick, 1988; Ghardirian, Gregoire, \& Kosmidis, 2000; Santosa et al., 2007; Schuldberg, 2001; Srivastava et al., 2010; Waddell, 1998). This is likely due to the number of eminently creative individuals who have been diagnosed with a mood disorder. Some of these people include Ernest Hemingway, Robert Lowell, Theodore Roethke, Virginia Woolf, Robert Schumann, Hugo Wolf, Jackson 
Pollack, Vincent Van Gogh, Edvard Munch, Arshille Gorky, Vivian Leigh, Dick Van Dyke, and Jason Robards (Andreasen \& Glick, 1988; Jamison, 1993; Rothenberg, 2001; Simonton, 1999). It is important to note that while some of the individuals listed above clearly had an affective disorder (e.g., Robert Schumann), many of these cases are conjectural and not based on modern clinical diagnoses. Furthermore, such compilations are not necessarily evidence of a link between creativity and mental illness. This is because lists can be generated even if the base rate of creators with psychopathology is much lower than that of the general population (Andreasen, 2008; Benham, 2005; Chavez-Eakle et al., 2006; Simonton, 1999; Waddell, 1998). Therefore, I have reviewed the empirical literature relating to the proposed relationship between creativity and the bipolar disorders from the perspective of a counseling psychology strength/asset focused perspective.

The bipolar disorders can be conceptualized as a spectrum of disorders. Some of the major demarcations of this continuum include a major depressive episode, dysthymia, euthymia, hyperthymia, hypomania, and mania. Hyperthymia, dysthymic disorder, and cyclothymic disorder represent distinct temperament types within this framework (Akiskal, 2004; Akiskal, Akiskal, Haykal et al., 2005; Akiskal, Mendlowicz, et al., 2005; Stahl, 2008). Temperament has traditionally been defined as aspects of personality that are heritable, stable, based on emotion, and not influenced by socio-cultural learning (Cloninger, 2004). Furthermore, it is important to note that within the bipolar spectrum of disorders framework, a hyperthymic temperament is not considered pathological

Some individuals who experience mild mood elevation (e.g., hypomanic episodes) perceive this experience to be conducive to their creativity (Andreasen, 2008; Jamison et al., 1980; Richards \& Kinney, 1990). At the same time, there are also individuals who have 
identified labile moods as impairing their creative efforts (Andreasen, 2008; Rothenberg, 2001). Discord remains whether conclusions regarding the potential association between facets of creativity and the bipolar disorders can be offered (Andreasen, 2008; Benham, 2005; ChavezEakle et al., 2006; Frantom \& Sherman, 1999; Ghadirian et al., 2000; Kinney \& Richards, 2007; Makel \& Plucker, 2008; Rothenberg, 2001; Waddell, 1998). It is my intention to consider both sides of this disagreement in critiquing the literature and to conduct a study that builds on the conclusions of this analysis.

There is accumulating empirical support from contemporary researchers that individuals who experience milder forms of the bipolar spectrum are more likely to be more creative than those who suffer from the more severe variants of the spectrum (Frantom \& Sherman, 1999; Ghardirian et al., 2000; Kinney \& Richards, 2007; Richards, 2001; Simonton, 1999; Waddell, 1998). It is important to emphasize that traits, rather than a full hypomanic episode, have been linked to aspects of creativity. Some authors have hypothesized that individuals diagnosed with cyclothymic disorder may possess a creative advantage because their moods are moderately intense relative to those who experience hypomania or mania (Akiskal \& Akiskal, 1988; Akiskal, Savino, et al., 2005; Andreasen, 2008; Richards, 2001; Simonton, 2002). Another possibility, which will be assessed by the current study, is that those who have a hyperthymic temperament may have a creative advantage over people at other points of the bipolar spectrum. There is a need for additional research that identifies how different groups of temperament/personality traits influence aspects of creativity (Chavez-Eakle et al., 2006; Mumford, 2003; Nowakoska, Strong, Santosa, Wang, \& Ketter, 2005).

The psychobiological model of personality development is based on the research of C. Robert Cloninger. Within this model, personality represents an evolving adaptation to both 
internal experience and the environment. Different variants of normal and abnormal personalities can be quantified in terms of four temperament trait dimensions and three character trait dimensions. These dimensions have been found to be independent and internally consistent. Temperament is purported to influence what an individual perceives while character modifies the meaning associated with these perceptions. A major assumption of the model is that susceptibility to most psychopathology is based on maladaptive personality (i.e., temperament/character configuration) development (Cloninger, 2004; Cloninger, 2006; Cloninger et al., 1997; Svrakic et al., 2002).

The seven dimensions of the psychobiological model of temperament and character interact with each other to facilitate adaptation to life experience as well as influence vulnerability to emotional and behavioral problems. Although dimensional, each trait can be defined in terms of dichotomies based on typical high and low scorers. The temperament dimensions include Harm Avoidance (anxious vs. risk taking), Novelty Seeking (impulsivity vs. stoical), Reward Dependence (gregariousness vs. aloofness), and Persistence (industriousness vs. underachieving). The character dimensions include Self-Directedness (responsible vs. externalizing), Cooperativeness (empathic vs. selfish), and Self-Transcendence (humble and fulfilled vs. proud and unfulfilled) (Cloninger, 2004; Cloninger et al., 1994).

The Temperament and Character Inventory - Revised (TCI-R) has been developed to assess the seven dimensions just described. It has demonstrated applicability to samples from both general and psychiatric populations. Specific configurations of the dimensions have evidenced different and consistent relationships to personality disorder subtypes, various other mental disorders (e.g., temperament styles that have been specified by the bipolar spectrum of disorder framework such as hyperthymia), patterns of brain activity, and unique genetic 
antecedents (Cloninger, 2004; Cloninger, 2008; Cloninger et al., 1994). Furthermore, hypotheses about temperament/character configurations that lead to creativity have been made but have yet to be extensively researched (Chavez-Eakle, Carmen Lara, \& Cruz-Fuentes, 2006).

It is noteworthy that the TCI-R produces configurations that account for individual differences in both normal and abnormal behavior patterns. As such, it assesses traits associated with pathology as well as qualities linked to life satisfaction (e.g., virtue, positive emotion) (Cloninger, 2004; Cloninger, 2008). This allows for the simultaneous investigation of the relationship between facets of creativity and positive as well as maladaptive characteristics. As such, it is a good measure to assess both pathological (e.g., cyclothymic temperament/disorder) and non-pathological (e.g., hyperthymic temperament) aspects of the bipolar spectrum.

A persistent and prevalent belief about the association between affective disorders and creativity is that severe mood swings are necessary in order to optimize the creative process (Akiskal \& Akiskal, 1988; Kinney \& Richards, 2007; Jamison, 1993; Plucker et al., 2004; Rothenberg, 2001). Some authors contend that such conceptions may factor into the decision of individuals diagnosed with a mood disorder to engage in behaviors that maintain their pathology (Benham, 2005; Chavez et al., 2006; Kinney \& Richards, 2007; Jamison, 1993; Plucker et al., 2004; Rothenberg, 2001). Likewise, uncritical acceptance of a possible relationship between affective disorders and creativity may impact how clinicians treat their clients such that they may unwittingly reinforce problematic behaviors (Benham, 2005). One way in which individuals may decide to maintain their creative abilities at the expense of their mental health is to decide not to take medications prescribed to them (Jamison, 1993; Lingam \& Scott, 2002). On an anecdotal level, I have worked with several clients who were diagnosed with a bipolar disorder and cited reduced creativity as a reason to not take the medication prescribed to them. 
Park, Levine, Weterman, Orfgen, and Foregger (2007) emphasize the importance of logically sound arguments and reasonable evidence when attempting to facilitate attitude change. Furthermore, they suggest that weak arguments can serve as a discounting cue that will produce change away from message recommendations. It is likely that providing individuals with information about the relationship between affective instability and optimal creativity would improve medication adherence. For those diagnosed with a bipolar disorder, such information could influence their beliefs and attitudes associated with taking medication. However, additional research is needed to support the aforementioned hypothesized relationship between affective instability and creativity so that the argument posed to these people will have a reasonable amount of evidence to support it.

In summary, the debate about the possible relationship between creativity and affective disorders has been longstanding. Despite disagreement regarding what conclusions can be made from earlier studies on the subject, contemporary literature suggests that at least some aspects of creativity may be enhanced by an affective range that is best characterized by the milder part of the bipolar spectrum of disorders. This part of the bipolar spectrum may be a hyperthymic temperament, which is not considered to be pathological. This leads to the research question and purpose of this dissertation: Are the creative advantages associated with the milder part of the bipolar continuum due to a specific type (or types) of temperament? The purpose of the proposed study is to begin addressing this important question.

The literature review below is comprised of four parts. First, the construct of creativity is discussed. Second, the psychobiological model of temperament and character is outlined. This model provides a theoretical framework for the proposed study. Third, the bipolar spectrum of disorders construct is reviewed. Fourth, research on the purported association between bipolarity 
and creativity is critiqued. Finally, conclusions about this literature review are presented and a study based on this discussion is proposed. 


\section{CHAPTER 2: Literature Review}

\section{The Construct of Creativity}

In this section creativity is identified as a multifaceted construct that is challenging to operationalize. The aspects of creativity that make it difficult to define are reviewed and various ways of accounting for its properties are discussed. Finally, the four historical ways of assessing the construct are delineated.

There is not a standard operational definition of creativity in the research literature (Andreasen, 2008; Benham, 2005; Feldhusen \& Goh, 1995; Plucker et al., 2004; Makel \& Plucker, 2008; Prentky, 2001; Mumford, 2003; Smith, 2005; Sternberg, 2006; Waddell, 1998). Empirical studies, program implementation, and prescriptive recommendations regarding creativity have all been performed without a clear conceptualization of the construct (Plucker et al., 2004; Makel \& Plucker, 2008; Waddell, 1998). It is commonplace for creativity to be utilized as a predictor or outcome variable without being defined (Plucker et al., 2004). Plucker et al. (2004) contend that use of a creativity measure is enhanced by an accompanying explanation of how it meets a definition of creativity identified by the researchers. However, researchers frequently have, "taken on the Herculean effort of legitimizing a program of research that is based on a construct that is implicitly understood and valued but explicitly defined in the most tenuous terms” (Plucker et al., p. 87).

There is disagreement regarding most aspects of creativity because it is a construct that incorporates many possible elements, which render it difficult to identify and define (Baer \& Feldhusen \& Goh, 1995; Kaufman, 2006; Sternberg, 2006). This is evident in the progression of creativity research. Whereas early authors attempted to designate one simple and universal 
mechanism to account for the construct (e.g., blind variation, divergent thinking, motivation, etc.), it is now commonly proposed that creativity is a complex phenomenon that consists of myriad influences. It is likely that many variables impact the creative process at different points, which includes the initial generation of an idea to the eventual generation of a creative product (Feldhusen \& Goh, 1995; Mumford, 2003; Simonton, 2002).

Creativity is also difficult to define because it is neither entirely domain specific nor domain general. Although the potential to be creative may entail some domain general elements, knowledge and skills specific to a particular domain are often required for one to make a creative contribution to a given field (Feldhusen \& Goh, 1995; Mumford, 2003; Simonton, 2002; Sternberg, 2006). For example, Mumford (2003) discussed how people in some vocations (e.g., artists, scientists, musicians) are frequently mentioned in the literature while others are rarely assessed (e.g., engineers, computer programmers, designers, advertising executives) despite the fact that all of these vocational areas necessitate creative invention to progress.

A second aspect of creativity research that contributes to the complexity of the construct is the distinction made between eminent creativity and everyday creativity, which has sometimes been referred to as big C and little c, respectively (Feldhusen \& Goh, 1995; Makel \& Plucker, 2008; Simonton, 2002). Eminent creativity has been investigated by some researchers who have asked leaders of a given field to nominate individuals who have made notable creative contributions. Others have identified a set of creative individuals from a recognizable group such as Nobel laureates (Andreasen \& Glick, 1988; Makel \& Plucker, 2008; Richards \& Kinney, 1990).

On the other hand, those who study everyday creativity investigate how individuals engage in creativity on a daily basis. These authors critique research that focuses on individuals 
who are considered eminently creative. They argue that although this effectively avoids the issue of having to define and measure the construct, it is problematic in that only an extreme subgroup of all creative people is being studied (Plucker et al., 2004; Richards, Kinney, Lunde, Benet, \& Merzel, 1988; Richards \& Kinney, 1990; Santosa et al., 2007; Smith, 2005).

Disagreement remains about whether everyday and eminent creativity are qualitatively or quantitatively distinct (Baer \& Kaufman, 2006; Feldhusen \& Goh, 1995; Richards, 2001; Simonton, 2002). If the two forms of creativity are qualitatively different, then each may have corresponding underlying personality attributes (e.g., level of susceptibility for psychopathology). However, if every day and eminent creativity differ quantitatively, then each would represent two parts of the same continuum and the same factors would be predictive of creative performance (Simonton, 2002).

A final reason why there is minimal agreement regarding creativity is because it has not been studied enough. Many scientists prefer to research phenomena that are more easily explored via traditional scientific analyses. Moreover, studies on creativity often take a long time to conduct, which can also serve as a deterrent for academics who must continue to publish in order to achieve satisfactory job performance. It is for these reasons creativity research has never been considered mainstream by either educational or psychological researchers, which further contributes to the unattractive prospect of making it the focus of an academic career (Sternberg, 2006).

Plucker et al. (2004) conducted a content analysis of creativity articles. They developed a protocol that assessed the frequency of the word creativity in each article, any direct or indirect definition(s) of the construct provided by the authors, and number of uses of the word before a definition was provided. Plucker et al. reviewed 30 articles in peer reviewed business, education, 
and psychology journals (10 articles in each area) that were dated from March, 1996 to August, 2002. The word creativity was included in the title of each article. Plucker et al. also reviewed 60 articles that were published in two major creativity journals (30 articles in the Creativity Research Journal and 30 articles in the Journal of Creative Behavior) between September, 1998 and January, 2002.

Plucker et al. (2004) stated that "of the 90 selected articles, only 34 (38\%) provided an explicit definition of the term creativity. Furthermore, 37 (41\%) provided an implicit definition, and 19 (21\%) provided no definition of the construct” (p. 88). The authors found that when researchers did offer an explicit definition of creativity, they often provided a wide range of definitions. It was likely that researchers were frequently discussing qualitatively different perspectives or facets of creativity (Plucker et al., 2004).

Plucker et al. (2004) offered an operational definition of creativity based on the reviewed literature. They stated “creativity is the interaction among aptitude, process, and environment by which an individual or group produces a perceptible product that is both novel and useful as defined within a social context” (p. 90). Plucker et al. suggested that aptitude includes creative abilities (e.g., tolerance for ambiguity, flexibility in thinking, perseverance), affective influences (e.g., attitude), and motivation. These aspects of creativity have been suggested by several other authors as well (e.g., Averill, 2002; Feldhusen \& Goh, 1995; Schuldberg, 1990; Strong et al., 2007; Srivastava et al., 2010). Plucker et al. also purported that creativity emerges from an interaction between these processes and environmental influences. While it was recognized that there are some unobservable qualities of the creative process, the authors indicated that some evidence of creativity (i.e., "perceptible product”) must exist for the construct to be evaluated (Plucker et al., 2004). 
Plucker et al. (2004) noted that novelty and usefulness were the most prevalent aspects of both explicit and implicit definitions of creativity in the literature. Many authors have identified novelty and usefulness as crucial to the definition of creativity (e.g., Andreasen, 2006; Averill, 2002; Caselli, 2009; Eysenck, 1995; Feist, 1999; Feist \& Barron, 2003; Mumford, 2003; Richards, 2001; Simonton, 2002; Sternberg, 2006; Waddell, 1998). The majority of these definitions have emphasized the necessity of a social context in determining whether (and how) a person, action, or product will be labeled creative (Andreasen, 2008; Plucker et al., 2004; Simonton, 1999; Smith, 2005). Plucker et al. hypothesized that including social context into the definition of creativity would cue researchers to specify the parameters of the construct in order to answer the question of "creativity for whom and in what context?" (p. 92).

A limitation of the Plucker et al. (2004) article is that art literature was not reviewed during the construction of the aforementioned operational definition of creativity. The notion that a creative product can be defined by how useful it is has been an ongoing subject of debate within this body of literature. The purposed dissociation between art and utility is epitomized by the French phrase "l'art pour l'art,” which translates to “art for art's sake.” This statement originates from Benjamin Constant who coined the phrase in 1804. It later began to become publically expressed via the lectures of Victor Cousin in Paris in 1817-1818. Essentially, Constant and Cousin argued that art should be free from ethical, political, religious, and/or moral scrutiny. Immanuel Kant further developed this line of thinking by suggesting that aesthetic judgment should not be associated with personal interests of any kind (Murphy, 2008).

Despite subtle disagreements on how art should be separated from the criterion of usefulness, the common theme of "art for art's sake" is maintained among individuals who question the purported association between creative products and utility (Murphy, 2008). There 
are also some writers within the art literature who suggest that art should have some type of usefulness. For example, Walter Benjamin suggested that a creative product should be judged on its ability to induce reflection and/or increase understanding in some capacity (Schwab, 2008).

There is no consensus regarding how creativity should be defined and/or operationalized. However, researchers in the educational and psychological fields continue to refine the definition of creativity by exploring its relationship with related constructs. For example, the complex association between creativity and intelligence continues to be elucidated (Baer \& Kaufman, 2006; Caselli, 2009; Mumford, 2003). Three conclusions about this relationship can currently be offered. First, creative individuals are likely to have IQ scores that are above average. Second, creativity is not necessarily enhanced by an IQ score that is higher than 120 and there is some evidence that extremely high IQ scores may be a detriment to creative performance (Baer \& Kaufman, 2006; Caselli, 2009; Feist \& Barron, 2003). Third, the IQ score and creativity association is dependent on what aspects of the constructs are being measured (Baer \& Kaufman, 2006).

There have been four historical ways to assess creativity. These divergent approaches are often referred to as person, process, product, and press (i.e., environment) (Baer \& Kaufman, 2006; Feldhusen \& Goh, 1995; Makel \& Plucker, 2008; Mumford, 2003; Simonton, 2002; Sternberg, 2006). The majority of creativity research utilizes instruments that assess person and/or process. This group of methods for measuring creativity exists because there is not a single or general theory of the construct to guide test developers (Feldhusen \& Goh, 1995; Simonton, 2002).

Numerous measures have been constructed to assess the relationship between personality characteristics and creativity. These instruments are typically based on the characteristics that 
have been associated with the construct. The assumption of this approach is that people who have personalities consistent with those who are creative will likely be creative themselves (Makel \& Plucker, 2008; Simonton, 2002). The most frequently utilized personality assessments rely on either self report or external ratings (e.g., independent judges, peers). Research regarding the validity of external ratings has been supported by some authors but refuted by others, which contributes to the ongoing controversy surrounding this form of assessment (Makel \& Plucker, 2008).

Biographical inventories are another way researchers have assessed the connection between personality and creativity. The assumption that underpins this manner of assessment is that prior creativity is the best predictor of future creativity. In addition, differences between creative and noncreative personalities may be influenced by variables such as family background, educational experiences, range of hobbies, and career activities (Makel \& Plucker, 2008; Simonton, 2002).

J.P. Guilford is often credited with providing impetus for scientific research of the creative process (Baer \& Kaufman, 2006; Makel \& Plucker, 2008; Mumford, 2003; Simonton, 2002; Sternberg, 2006). His investigation of creativity and problem solving derive from a portion of the Structure of Intellect model he proposed in 1967. Essentially, Guilford depicted the construct of intelligence as a cube with the dimensions of operations (i.e., thought processes), contents to which operations could be applied, and products that might result from the performance of operations in divergent content areas. Different types of operations include cogitation, memory, divergent production, convergent production, and evaluation. The divergent production operation eventually became linked to creativity research by Guilford’s Unusual Uses Test, which measures divergent thinking (Baer \& Kaufman, 2006; Makel \& Plucker, 2008). 
The Torrance Tests of Creative Thinking (TTCT) were based on Guildford's work and eventually became the "bedrock of the psychometric assessment of creativity" (Makel \& Plucker, 2008, p. 249). This instrument is purported to measure testing fluency (i.e., total number of responses), flexibility (i.e., number of different kinds of responses), originality (i.e., the uniqueness and rarity of response), and elaboration (i.e., the detail of responses). This measure has been utilized so often that many lament how creativity has often been viewed as synonymous with divergent thinking (e.g., Baer \& Kaufman, 2006; Mumford, 2003; Sternberg, 2006). Although the TTCT has been revised several times and remains popular, it has also been criticized because divergent thinking is a gauge of novelty but not necessarily usefulness. Furthermore, several authors suggest that creativity research has become overly reliant on divergent thinking tests to the exclusion of other assessment methods (Feldhusen \& Goh, 1995; Makel \& Plucker, 2008; Mumford, 2003; Simonton, 2002).

The criticisms of the TTCT mentioned above are reminiscent of one of the points made by Baer and Kaufman (2006) in regard to the relationship between IQ and creativity. Specifically, whether or not an association is found between creativity and intelligence depends on what aspects of creativity are measured. This is likely to be the case for the purported link between creativity and any other variable (e.g., affect). Some researchers use product assessments to measure creativity and avoid the aforementioned concerns regarding external ratings and divergent thinking tests (Baer \& Kaufman, 2006; Makel \& Plucker, 2008; Simonton, 2002). Although some rating systems to judge creative products have been offered, none of these approaches have been able to establish criterion validity since there are no universally accepted standards for assessing creativity. Furthermore, there are many methods for establishing who is 
qualified to judge creative products (e.g., the skill of the subjects and/or judges, the target domain, the purpose of assessment) (Makel \& Plucker, 2008).

The impact of how environment influences what is considered creative is becoming increasingly recognized as another aspect of the construct (Baer \& Kaufman, 2006; Makel \& Plucker, 2008 Mumford, 2003). Most of the research in this area has focused on comparisons between personality traits, creative product ratings, and environmental factors. However, a psychometrically acceptable measure of environmental influence on creativity has yet to be established. Moreover, specific environmental variables that facilitate/inhibit creativity need to be determined (Makel \& Plucker, 2008).

Biographical methods and shared-trait research are two ways in which the possible association between creativity and mental illness has been investigated. Potential diagnosable conditions in deceased eminent creators have been assessed via biographical methods. In these studies, creativity is often defined by the person's achieved fame, granted by virtue of a recognized achievement, and/or participants are identified as creative by those familiar with their work (e.g., mentors, teachers, peers, etc.). Psychopathology is retrospectively assessed by categorical diagnosis, the presence of specific symptoms, and/or scores on psychological tests (Schuldberg, 2001).

There are several limitations to these retrospective studies of biographical information. First, psychopathology is identified via reports of symptoms or material the deceased has produced (e.g., a diary) and often is not independently verified. As such, this information may not be complete or accurate. Second, the diagnostic criteria that are utilized are often not well defined and/or vary across different studies. Third, individuals in this research are infrequently compared to controls so it cannot be determined how (or if) their histories differentiate them 
from less eminent creators whose backgrounds are unknown. Finally, eminence can be as difficult to operationally define as creativity (Andreasen, 2008; Benham, 2005; Prentky, 2001; Richards \& Kinney, 1990; Rothenberg, 2001; Santosa et al., 2007; Schuldberg, 2001; Waddell, 1998). Despite the aforementioned limitations of this approach, retrospective biographical studies are often provided as evidence of the hypothesized link between creativity and psychopathology (Benham, 2005; Rothenberg, 2001; Waddell, 1998). As such, the robustness of this purported association can be questioned. Moreover, alternative ways of conceptualizing the relationship between creativity and psychopathology (e.g., creativity manifests from psychological strength regardless of the presence of mental illness) should be considered.

In shared-trait research, psychometric assessment is conducted to identify characteristics that are related to creativity. This has been accomplished in three different ways. First, groups of professional artists have been compared to control groups of individuals who are not artists. Second, measures of creativity and psychopathology have been utilized to define groups within a sample of participants and then these groups are compared. Third, creativity measures have been administered to a sample from a psychiatric population (Benham, 2005; Schuldberg, 2001).

One benefit of the shared-trait approach is that it can highlight both common and discrepant elements of creativity and psychopathology. This allows for the possibility of creativity being associated with positive, healthy traits that may serve to actually mitigate the impact of coexisting problematic symptoms. A drawback of past shared-trait research is that it has largely been conducted in the absence of a guiding theory that accounts for what characteristics are linked to creativity (Schuldberg, 2001).

There is accumulating evidence that associates heritable components of temperament and personality traits to susceptibility for psychiatric disorders (Cloninger, 2004; Cloninger, 2006; 
Nowakowska, Strong, Santosa, Wang, \& Ketter, 2005). Additional research that identifies how groups of temperamental traits influence aspects of creativity is needed (Mumford, 2003; Nowakoska et al., 2005). Many personality researchers purport that variance in personality can be accounted for by five to seven personality dimensions. Factor analyses have consistently demonstrated this range of dimensions despite the theoretical differences between the different personality models that are being studied (Faber, 2003).

\section{The psychobiological model of temperament and character}

This section is comprised of several parts. First, the evolution of the psychobiological model of temperament and character is reviewed. Second, the constructs of temperament and character are distinguished and defined. Third, the four dimensions of temperament and three dimensions of character are discussed. Fourth, the relationship of temperament and character to the concept of personality is underscored. Fifth, the applicability of the model to both normal and abnormal populations is highlighted. Finally, research about how temperament/character configurations were established is provided.

The psychobiological model of personality development is based on the research of C. Robert Cloninger. Initially, he identified three dimensions of temperament (i.e., Harm Avoidance, Novelty Seeking, Reward Dependence) from his review of prior studies on genetic structure of personality, neurobiological studies in humans and other animals, and behavior conditioning. Temperament is defined as the emotional core of personality and is thought to constitute "heritable neurobiological dispositions to early emotions (such as fear, anger, and attachment), and their related automatic behavior reactions (such as inhibition, activation, and maintenance of behavior) in response to specific environmental stimuli (danger, novelty, and reward, respectively)” (Svrakic et al., 2002, p. 190). Thus, temperament represents what a person 
focuses his or her attention on and how intensely he/she responds to stimuli (Cloninger, 1987; Cloninger, 2004; Svrakic, Svrakic, \& Cloninger, 1997).

The Tridimensional Personality Questionnaire (TPQ) was developed to measure the hypothesized dimensions of temperament. Factor analytic studies confirmed that these dimensions were significantly correlated and internally consistent once a fourth dimension (i.e., Persistence) was created. The four temperament dimensions have been the subject of clinical, neurobiological, and genetic research. Each has been found to be approximately 50 percent heritable according to twin studies and generally demonstrate minimal change with mood state or increasing age. The structure and stability of these temperament dimensions has been replicated in several different cultures via the use of different translations of the TPQ. The same temperament dimensions can be assessed regardless of age via the use of age-appropriate questions (Cloninger, 2004; Cloninger et al., 1994; Goncalves \& Cloninger, 2010).

Individuals who score high on Harm Avoidance are likely to be characterized as cautious, careful, fearful, tense, apprehensive, anxious, timid, doubtful, discouraged, insecure, passive, negativistic, and/or pessimistic. They are often inhibited in most social situations and generally have a low energy level. These people are typically hypersensitive to criticism and punishment. In contrast, low scorers on Harm Avoidance have a tendency to be carefree, relaxed, daring, courageous, composed, and optimistic. They are likely to be socially confident and demonstrate a high energy level (Cloninger et al., 1994).

People who score high on Novelty Seeking are frequently described as quick tempered, excitable, exploratory, curious, enthusiastic, exuberant, easily bored, impulsive, and disorderly. They tend to be strongly attracted to the new and unfamiliar, which leads to exploration of potential rewards. However, their relationships are often unstable due to their frequent irritability 
and quick disengagement when their desires are thwarted. On the other hand, individuals who score low on Novelty Seeking are likely to be even tempered, indifferent, unenthusiastic, stoical, reflective, frugal, reserved, accepting of monotony, systematic, and orderly (Cloninger et al., 1994).

Individuals who score high on Reward Dependence are often characterized as sympathetic, loving, warm, sensitive, dedicated, dependent, and sociable. They tend to openly communicate with others and actively seek interpersonal interaction. In addition, these people are typically sensitive to social cues but can also be easily influenced by others. In contrast, those who score low on Reward Dependence are frequently practical, stubborn, cold, and interpersonally insensitive. They are likely to be satisfied with being alone and prefer to maintain a certain degree of interpersonal distance (Cloninger et al., 1994).

People who score high on Persistence are likely to be industrious, hard-working, ambitious, overachieving, and stable despite frustration or fatigue. They are often eager to begin work and frequently intensify their efforts in response to an anticipated reward. In addition, these individuals are likely to perceive frustration as a personal challenge and tend to intensify their efforts when they are criticized or confronted with mistakes. On the other hand, those who score low on Persistence are often characterized as inactive, unreliable, satisfied with their current accomplishments, underachieving, and erratic. These individuals infrequently make more effort in anticipation of reward and are typically slow to begin work. In addition, these people are likely to quit when they encounter frustration, criticism, obstacles, and/or fatigue (Cloninger et al., 1994).

Cloninger and his colleagues discovered that all of the possible temperament combinations were found to occur in both individuals who were diagnosed with a personality 
disorder and those who did not, even though the probabilities of each configuration differed. As a result, three character dimensions were derived from comparisons of the TPQ with other personality measurement systems as well as descriptions of character (Cloninger, 2004; Cloninger, 2008; Cloninger et al., 1994).

Character is hypothesized to "optimize adaptation of early temperament to the environment by modulating the salience of early percepts and affects regulated by temperament, thus reducing the maladaptive impact of 'raw' temperamental traits” (Svrakic et al., 2002, p. 191). In contrast to temperament, character is moderately influenced by social learning and cultural expectations regarding personal roles. It matures in a stepwise manner that occurs in incremental shifts from early life to late adulthood. Thus, character is conceptualized as a conglomerate of personal goals, values, and traits that can be specified in terms of subject-object relations. These subject-object relations impact an individual's voluntary choices, intentions, and meaning of different experiences (Cloninger, 2004; Cloninger et al, 1994; Svrakic, Svrakic, \& Cloninger, 1996).

The subject-object distinction provides three possible relationships; the self (i.e., subjectsubject), interactions with others (i.e., subject-object), and participation in the world as a whole (i.e., object-object). Three character dimensions were created to reflect each of these relationships and include Self-Directedness (self-concept), Cooperativeness (concept of interactions with others), and Self-Transcendence (concept of one's participation in the world). These scales were added to the TPQ to create the Temperament and Character Inventory (TCI). Thus, the TCI is comprised of four temperamental trait dimensions (i.e., Harm Avoidance, Novelty Seeking, Reward Dependence, and Persistence) and three character trait dimensions 
(i.e., Self-directedness, Cooperativeness, and Self-transcendence) (Cloninger, 2004; Cloninger et al., 1993; Cloninger et al., 1994).

Individuals who score high on Self-Directedness are often characterized as mature, strong, self reliant, responsible, goal oriented, and constructive. They generally have high self esteem and are able to adapt their behavior according to their independently identified goals. In contrast, people who score low on Self-Directedness tend to be immature, weak, fragile, blaming, destructive, ineffective, irresponsible, and unreliable when they are not under the direction of a leader. They generally lack an internal organizational principle, which makes it difficult for them to define and pursue meaningful goals. As such, they generally react to stimulation and pressure from external circumstances (Cloninger et al., 1994).

Individuals who score high on Cooperativeness are likely to be described as empathetic, tolerant, compassionate, supportive, fair, and principled. They generally understand and respect the needs of others. On the other hand, those who score low on Cooperativeness are likely to prefer solitude and experience difficulty with interpersonal interaction (Cloninger et al., 1994).

People who score high on Self-Transcendence are often characterized as humble, fulfilled, patient, selfless, creative, and spiritual. They tend to be tolerant of ambiguity and uncertainty. In addition, they are generally thankful for both their successes and failures. In contrast, individuals who score low on Self-Transcendence are typically proud, impatient, unimaginative, materialistic, and unfulfilled. They are often intolerant of ambiguity and uncertainty. As a result, these people frequently have a need for control and experience difficulty with being satisfied with what they have achieved (Cloninger et al., 1994).

The TCI has demonstrated applicability to samples from both the general and psychiatric populations. Specific configurations of the dimensions have evidenced different and consistent 
relationships to personality disorder subtypes, various other mental disorders (e.g., anxiety disorders, mood disorders, eating disorders, etc.), patterns of brain activity, and unique genetic antecedents. In summary, the seven dimensions interact with each other to facilitate adaptation to life experience as well as influence susceptibility to emotional and behavioral problems (Cloninger, 2004; Cloninger, 2008; Cloninger et al., 1994).

Traditional subtypes of temperament and personality disorders correspond to TCI temperament configurations of Harm Avoidance, Novelty Seeking, and Reward Dependence. Persistence is thought to be a general moderator of self-control. It influences character development by contributing to emotional regulation and organization of personality. However, Persistence is not included in the actual configurations of temperament and personality disorder subtypes. While different temperament configurations are associated with differing risks of particular behaviors (e.g., violence, charity, etc.), the accuracy of predictions about individual behavior is low unless character configurations are also specified. High scores on all three character dimensions have been strongly associated with resilient positive emotions, satisfying quality of life, and virtuous conduct. As such, the measure can generate profiles that are able to account for individual differences in both normal and abnormal behavior patterns (Cloninger, 2004; Cloninger, 2008; Cloninger et al., 1994; Cloninger et al., 1997).

The interpretation of temperament and character configurations for both clinical and nonclinical populations is based on where an individual's scores fall on a range of cut off points. The demarcations are based on percentiles and include very low (0-16.7\%), low (17-33\%), average (34-66\%), high (67-83\%), and very high (84-100\%). It is commonplace in research studies for the "very low" and "low" as well as the "high and "very high" groups to be combined. As such, configurations are based on where a participant scores in one of three percentile ranges (i.e., 
lowest one third, 0-33\%, middle one third, 34-66\%, and highest one third, 67-100\%). These categories have been found to be the most reliable in analyses of the TCI. Thus, the TCI is both dimensional and categorical. The instrument assesses where a person fits on each of the seven dimensions and provides a composite configuration of these scores that can be described categorically (Cloninger et al., 1994).

The combination of temperament and character underpins personality (Cloninger, 2006; Svrakic et al., 2002). In this model, personality is defined as "the dynamic organization within the individual of those psychobiological processes by which an individual uniquely adapts to changing internal and external environments” (Cloninger, 2006, p. 69). In other words, personality represents an evolving adaptation to both internal experience and the environment. It includes systems that regulate cognition, emotions, mood, impulse control, and social interaction. Thus, personality traits are enduring patterns of perception, relation, and thought about oneself, others, and the world as a whole (Cloninger, 2004; Cloninger, 2006; Cloninger et al., 1997).

In summary, temperament is purported to influence what an individual perceives while character modifies the meaning associated with these perceptions. Temperament constrains character but does not completely determine it due to the effects of socio-cultural learning and experience. Therefore, the feelings, thoughts, and behaviors of different people are complex and unique. However, personality can be viewed as lawful to the extent that it can be reliably quantified. As a result, predictions about personality dynamics can be made based on relationships between its constituent parts (Cloninger, 2004; Cloninger, 2006; Cloninger, Svrakic, \& Svrakic, 1997; Svrakic et al., 1996; Svrakic et al., 2002).

Temperament and character can further be distinguished by the different types of learning to which each corresponds. Temperament entails the basic processes of sensation, association, 
and emotion that collectively manifest individual differences in the conditioning of habits and skills (i.e., procedural learning). It represents a composite of multiple emotional drives that can be both conflicting and synergistic. Temperament configurations "specify a set of automatic response patterns that represent an implicit model of expectations about what is adaptive or satisfying to an individual” (Svrakic et al., 1996, p. 253). In contrast, character is comprised of logic, evaluation, and abstraction that collectively create individual differences in concept-based processing of goals and values (i.e., propositional learning). The implication of these two types of learning is that "as character develops, the direction of personality development may shift from being primarily steered by passive responses regulated by heritable temperament to being steered predominantly by active symbolic constructs regulated by socially molded character traits” (Svrakic et al., 1996, p. 258).

The complex patterns of relationships between temperament, character, and the types of learning associated with each can be quantified by the TCI as a nonlinear dynamic network of variables. In other words, the effect of each variable is dependent on the other variables with which it is combined. As such, personality is nonlinear and manifests qualitatively changeable interactions among different components that are etiologically distinct. In essence, personality represents the pattern of change between the three character dimensions as a function of the four temperament dimensions, genetic factors, family environment, socio-cultural norms, and random environmental events. There is not a one-to-one relationship between temperament and character configurations as would be expected by a linear system. Each temperament configuration can develop into multiple character configurations (i.e., multifinality). In a similar vein, different temperament configurations may develop into the same character configuration (i.e., 
equifinality) (Cloninger, 2004; Cloninger, 2006; Cloninger, Svrakic, \& Svrakic, 1997; Svrakic et al., 2002).

The dimensions of temperament have been shown to be relatively stable over time and to be universal across various cultures with different political and ethnic groups (Cloninger, 2006; Svrakic et al., 2002). In contrast, character traits change with age and psychological maturation such that "the final character outcome can be predicted as a non-linear function of antecedent temperament traits, socio-cultural pressures, and random life events unique to the individual” (Svrakic et al., 2002, p. 191). It has been found that the heritability of character is equivalent to that of temperament but character has been associated with more recently evolved parts of the brain than temperament (Cloninger, 2008).

Abnormal personality development is thought to underlie nearly all psychopathology with the exception of brain function defects attributable to medical conditions. This proposition is based on three hypotheses. First, personality can be quantitatively specified as a selforganizing multidimensional complex. Second, susceptibility to most psychopathology is based on problematic personality development. Third, personality and its associated disorders can be quantitatively described in terms of the aforementioned nonlinear adaptive system (Cloninger et al., 1997).

There are two additional tenets of the psychobiological model of temperament and character that are important to highlight. First, the model is based on the finding that individual genetic and psychological variables have an approximately equal impact on personality. Second, the complete range of personality is defined by traits that contribute to various difficulties (e.g., personality disorders, depression, etc.) as well as traits that are indicative of life satisfaction (e.g., virtue, positive emotion) (Cloninger, 2004). 
Specific pairings of the temperament and character dimensions into configurations were established in a sample of 593 individuals from the general population. In addition, a one year follow-up study of this sample found that of the individuals who demonstrated character configuration changes (i.e., 121 of 593 participants), 96\% changed in a specific direction predicted by the nonlinear dynamic model. In other words, the change that occurred in an individual's character configuration was the most probable alternative configuration predicted by the individual's identified temperament configuration. In summary, the combination of temperament and character configurations, "allows a quantitative prediction of transitions in configuration over time that is sensitive to quantitative differences in multiple personality dimensions simultaneously (the internal milieu) as well as to external influences, such as social learning and random events” (Cloninger et al., 1997, p. 891-892). Specific temperament and character configurations germane to this dissertation will be discussed later.

\section{The bipolar spectrum of disorders}

The bipolar spectrum of disorders construct is discussed in this section. First, the definition of mood disorders is reviewed and aspects of the proposed bipolar continuum are delineated. Second, characteristics of this model germane to this dissertation (i.e., temperament, sub-threshold symptoms) are introduced. Third, research on the differences between the bipolar spectrum of disorders and how the bipolar disorders have been classically defined is provided.

Mood disorders are often referred to as affective disorders because affect is the external manifestation of mood or emotion (Stahl, 2008). Emotional states manifest from an individual's personality, which can be defined in terms of traits. As a result, affect can be considered as a state or trait (Averill, 2002; Stahl, 2008). Depression and mania are viewed as opposite ends of

the mood spectrum that have traditionally been conceptualized as different "poles” (Stahl, 2008, 
p. 454). Thus, mood disorders are sometimes categorized as either unipolar (i.e., individuals who only experience depressive episodes) or bipolar (i.e., individuals who experience some aspect of the manic pole). Affective experience can also be thought of as dimensional. Some of the major parts of the continuum include a major depressive episode, dysthymia, euthymia, hyperthymia, hypomania, and mania (Stahl, 2008).

The most prevalent mood disorder is major depressive disorder, which is diagnosed by the incidence or recurrence of depressive episodes (Stahl, 2008). Dysthymic disorder is a less severe but more persistent form of depression. The primary feature of bipolar disorder is episodes of disturbed mood that meet criteria for mania (bipolar I disorder) or hypomania (bipolar II disorder) (American Psychiatric Association, Diagnostic and Statistical Manual of Mental Disorders, 2000; Stahl, 2008). Studies indicate five to 15 percent of individuals who have a hypomanic episode will eventually have a manic episode as well. The simultaneous occurrence of depressive and manic symptoms for a one week period is termed a mixed episode (DSM-IVTR, 2000; Stahl, 2008).

Cyclothymic disorder is characterized by several periods of hypomanic symptoms as well as periods of depressive symptoms that do not meet the criteria for a major depressive episode (DSM-IV-TR, 2000, p. 396). Individuals diagnosed with this disorder can experience a depressive temperament (i.e., below euthymic mood but does not meet the threshold for a mood disorder) and/or a hyperthymic temperament (i.e., above euthymic mood but does not meet the threshold for a mood disorder) (Stahl, 2008). Some believe cyclothymic disorder should be conceptualized as a distinct type of temperament (Akiskal, 2004; Akiskal, Akiskal, Haykal, Manning, \& Conner, 2005; Akiskal, Mendlowicz, et al., 2005; Stahl, 2008). 
Some authors refer to the bipolar disorders and cyclothymic disorder as the bipolar spectrum of disorders. Dysthymic disorder and hyperthymia represent temperament types within this framework (Akiskal, 2004; Akiskal, Akiskal, Haykal et al., 2005; Akiskal, Mendlowicz et al., 2005; Bader \& Dunner, 2007; Judd \& Akiskal, 2003; Smith, Ghaemi, \& Craddock, 2008; Stahl, 2008). This alternative conceptualization of the bipolar disorders was first proposed in 1977 and research has gradually enriched it with conceptual extensions, modifications, and empirical support (Akiskal, 2004; Smith et al., 2008). For example, one consistent finding has been an elevated prevalence of the milder disorders in relatives of individuals diagnosed with bipolar disorder I (BPD I) (Kinney \& Richards, 2007; Richards \& Kinney, 1990).

The bipolar spectrum of disorders is linked to a variety of symptoms such as early age of onset, high recurrence (i.e., at least five episodes), rapid onset and offset of episodes, postpartum depression, marked seasonality, atypical features (i.e., hypersomnia and overeating), hostile and/or labile mood, and isolated hypomanic symptoms during depression. Furthermore, additional associated variables include cycling on antidepressants, refractoriness (i.e., at least three antidepressants from different classes), family history of bipolar disorder, and family history of a mood disorder in three generations (Akiskal, 2004; Smith et al., 2008).

There is also developing recognition that many individuals may experience problematic moods that do not qualify for one of the specific disorders listed in the DSM-IV (Akiskal, 2004; Bader \& Dunner, 2007; Judd \& Akiskal, 2003). Moreover, it has been estimated that approximately 25 to 50 percent of people diagnosed with recurrent unipolar depression may be better classified as having a bipolar spectrum disorder (Akiskal, 2004; Judd \& Akiskal, 2003; Allen \& Smith, 2008; Smith et al., 2008). As such, many people have often been given diagnoses 
of mood disorder not otherwise specified or bipolar disorder not otherwise specified (Akiskal, 2004; Bader \& Dunner, 2007; DSM-IV-TR, 2000; Stahl, 2008).

Stahl (2008) indicated that the current diagnostic system has created “a huge single category for many patients that belie the richness and complexity of their symptoms. Increasingly, such patients are seen as belonging in general to the 'bipolar spectrum' and, in particular, to one of several additional descriptive categories” (p. 461). Several authors believe that the reformulation of bipolar disorders as a spectrum of disorders would have clinically meaningful implications for comorbidity, clinical management, and public health (Akiskal, 2004; Bader \& Dunner, 2007; Judd \& Akiskal, 2003; Smith et al, 2008; Stahl, 2008). Such revisions would attend to a group of individuals who experience various admixtures of depression, hypomania, and temperamental instability (Akiskal, 2004; Smith et al., 2008; Stahl, 2008). Moreover, this subgroup of people is likely to have been diagnosed with a unipolar depression, which may account for several inconsistencies in the biological studies of this disorder (Smith et al., 2008).

There are two types of mood disorder that have been referred to as bipolar .25 and bipolar .5 despite the fact that these labels are not recognized by the American Psychiatric Association (Stahl, 2008). Bipolar .25 “could designate an unstable form of unipolar depression that responds sometimes rapidly but in an unsustained manner to antidepressants” (Stahl, 2008, p. 461). These individuals experience unstable affect and can sometimes benefit from taking mood stabilizer treatments in addition to an antidepressant. Bipolar .5 is essentially what is currently viewed as schizoaffective disorder (Stahl, 2008).

The bipolar spectrum of disorders may include individuals who experience protracted or recurrent hypomania without any evidence of a depressive episode (Kinney \& Richards, 2007; 
Stahl, 2008). It is common for these people to eventually experience a major depressive episode, which would then qualify them for a bipolar disorder II (BPD II) diagnosis. They are typically treated with mood stabilizers and an antidepressant medication is considered if these patients develop symptoms of depression (Stahl, 2008).

There are some people who develop symptoms of mania or hypomania subsequent to undergoing various forms of treatment such as antidepressant medications, electroconvulsive therapy, light therapy, or medication prescribed for other general medical conditions (Akiskal, 2004; Smith et al., 2008; Stahl, 2008). These people are thought to have a diathesis for a disorder in the bipolar spectrum. They are at increased risk for developing future manic or hypomanic episodes regardless of whether they cease the treatment that provided impetus for their symptoms (DSM-IV-TR, 2000; Stahl, 2008). This condition has been referred to as bipolar III disorder (Akiskal, 2004; Richards \& Kinney, 1990; Stahl, 2008) but is not a diagnosis identified by the American Psychiatric Association (DSM-IV-TR, 2000). These individuals are not good candidates for antidepressant monotherapy (Allen \& Smith, 2008; Smith et al., 2008; Stahl, 2008).

People who experience depressive episodes with a preexisting hyperthymic temperament are thought to have bipolar IV disorder (Akiskal, 2004; Stahl, 2008). Hyperthymic temperament (which is synonymous with the term hyperthymia) is distinguished from hypomania because it represents an individual's habitual baseline rather than manifesting as distinct episodes (Akiskal, 2004). Hyperthymia can be diagnosed when four or more of the following attributes are present as part of a person's habitual long-term functioning; upbeat and exuberant, articulate and jocular, overoptimistic and carefree, overconfident and boastful, high energy level, versatile with broad interests, over-involved and meddlesome, uninhibited and risk-taking, and short sleeper (i.e., less 
than six hours nightly). There is evidence that these traits often occur in some individuals who are diagnosed with major depressive disorder and have a family history of bipolar disorder (Akiskal, 2004; Kinney \& Richards, 2007). Although this is not a formally recognized bipolar disorder, these people may respond best to mood stabilizers. They may experience rapid cycling, hypomania, or mixed states if they only take an antidepressant (Stahl, 2008).

There are many additional possible subtypes of mood disorder that could be included within the bipolar spectrum of disorders. Akiskal (2004) contends that a common feature among many people with conditions that can be considered part of the middle of the spectrum (i.e., those with disorders between BPD I and unipolar depression) is the experience of hypomania at a subthreshold level. This specific range is referred to as the soft bipolar spectrum (Akiskal, 2004).

The discrepancy between DSM-IV-TR criteria and clinical guidelines regarding the diagnosis of a hypomanic episode can be utilized to delineate what is meant by the term “subthreshold.” Specifically, clinical guidelines suggest that when numerous hypomanic episodes have occurred in the past, the duration of these periods is not crucial to rendering the diagnosis (Akiskal, 2004; Smith et al., 2008). However, a hypomanic episode is defined by the DSM-IV-TR as having lasted at least four days, which qualifies any occurrence of hypomania that does not persist for this time period as subthreshold (Akiskal, 2004; Allen \& Smith, 2008). There is accumulating evidence that the median duration of hypomanic symptoms in those diagnosed with a bipolar disorder is between one and three days (Allen \& Smith, 2008; Stahl, 2008).

Judd and Akiskal (2003) investigated the long term symptom status of a large clinical sample of individuals diagnosed with either BPD I or BPD II. The symptoms in this sample were found to frequently fluctuate in terms of polarity and severity. Furthermore, the authors found 
that the long term symptomatic course of these individuals was often dominated by moderate and subsyndromal affective symptoms rather than episodes of mania or major depression. Judd and Akiskal concluded that the longitudinal symptomatic course of bipolar disorder is dimensional rather than categorical. Moreover, they suggested that any level of observed affective symptoms indicated that a bipolar illness is likely present and active (Judd \& Akiskal, 2003).

Judd and Akiskal (2003) identified how critics of the proposed bipolar spectrum of disorders have often cited findings from the US Epidemiological Catchment Area (ECA) database to argue for the relative rarity of bipolar disorder. Specifically, critics have maintained that bipolar disorder has been estimated to impact $1.3 \%$ of the population. In their view, this indicated that the much higher estimates of bipolarity, which have been provided by other researchers, were inaccurate because the results did not derive from national data. Judd and Akiskal acknowledged how the majority of research on bipolar disorder has investigated variables such as chronicity and suicide in follow-up studies but the incidence of the proposed bipolar spectrum has not been assessed in a large scale study (Judd \& Akiskal, 2003).

Judd and Akiskal (2003) conducted a secondary analysis of the ECA household data and made two predictions. First, they hypothesized the aggregate of lifetime prevalence of subsyndromal, hypomanic, and manic symptoms would exceed that which has been previously reported. Second, they purported that when compared to participants with no mental disorders, each level of bipolar symptom severity (including subsyndromal manic/hypomanic symptoms) would be associated with significantly elevated lifetime health services use, need for welfare and disability benefits, and suicidal behavior (Judd \& Akiskal, 2003).

Judd and Akiskal (2003) reviewed how diagnoses were rendered at the time of data collection. Participants were administered the Diagnostic Interview Schedule (DIS) by lay 
interviewers. These interviews were recorded and then assigned diagnoses by a computerassisted algorithm. The authors did not discuss if the lay interviewers received any type of training in administering the DIS. Judd and Akiskal should have provided more details about how the computer determined diagnoses based on an algorithm.

Judd and Akiskal (2003) indicated that the DIS specifies a standardized threshold for determining if a manic symptom is clinically relevant. It stated the symptom must be present "for at least 1 week, with respondents being so happy, excited or high that they got into trouble, or their family or friends were worried about it, or a doctor said they were manic in need of psychiatric treatment” (p. 125). The authors reclassified all of the participants of the original ECA household sample into four mutually exclusive categories based on the presence/absence and severity of manic symptoms or episodes. They were assessed in terms of lifetime prevalence, which was what had been measured during the initial ECA interview. The new groups included manic episode (i.e., had a diagnosis of a manic episode), hypomanic episode (i.e., had a diagnosis of a hypomanic episode but not a manic episode), subsyndromal symptoms of mania (i.e., experienced two or more manic symptoms without meeting the full criteria for a hypomanic episode or manic episode), and comparison group (i.e., no mental disorder or manic spectrum symptoms).

Judd and Akiskal (2003) performed a logistic regression analysis to examine the association between the aforementioned diagnostic groups and a series of psychosocial dependent variables. The dependent variables were specified and categorized as service utilization for emotional/substance abuse/mental health problems, participation in public assistance programs, and suicidal ideation/behavior. The authors then calculated odds ratio estimates, which they described as a measure of association between the three disordered groups 
(i.e., mania, hypomania, subsyndromal symptoms of mania) and the dependent variables. Odds ratios estimate the relative risk of the presence of a dependent variable that can be associated with one diagnostic group in comparison with the others. Group differences in regard to age, gender, race/ethnicity, and study site were controlled (Judd \& Akiskal, 2003).

Judd and Akiskal (2003) found that there was a 5.1\% lifetime prevalence rate of subthreshold manic/hypomanic symptoms in the ECA data, which was consistent with the prevalence rates reported by studies that have investigated the prevalence of such symptoms in smaller clinical samples. Furthermore, subthreshold manic/hypomanic symptoms were almost four times more common than the combined prevalence rate of hypomanic and manic episodes (1.3\%). The total lifetime prevalence was over $6 \%$ whereas the previous analysis had reported a prevalence rate of $1.3 \%$. The authors concluded their findings supported the notion that bipolar disorders are dimensional and constitute a range of symptom severity. Moreover, their results revealed that past epidemiological surveys have underestimated the prevalence of bipolar diathesis in the population due to an exclusive focus on manic and/or hypomanic episodes. This proposition has been echoed by other researchers as well (Allen \& Smith, 2008; Smith et al., 2008).

Judd and Akiskal (2003) discovered that in comparison to the group with no mental disorder, the group of participants who demonstrated subsyndromal symptoms of mania was associated with significant increases in health services use, need for public assistance, and suicidal behavior. Thus, they suggested "the subsyndromal manic symptoms are a clinically relevant, integral component of the longitudinal symptomatic picture of bipolar disorders and as such should be included when estimating the lifetime prevalence of the bipolar diathesis in any sample studied" (p. 129). 
Judd and Akiskal (2003) provided convincing evidence for the existence of the bipolar spectrum of disorders. They used the conceptualization of subsyndromal manic symptoms to reanalyze data from a large scale study that had previously been employed to question both the incidence and importance of manic subsyndromal symptoms. In doing so, the authors produced lifetime prevalence rates that were akin to what has been reported by smaller studies.

Furthermore, people who experienced subsyndromal symptoms of mania exhibited significantly more difficulties than those who do not experience any level of such symptoms (Judd \& Akiskal, 2003). One implication of these findings is that much of the past research on the putative association between bipolarity and creativity is flawed to the extent that it assesses some aspects of the bipolar spectrum of disorders (e.g., BPD I, BPD II) but excludes more subtle parts of the bipolarity continuum (e.g., sub-syndromal symptoms of hypomania, different types of temperament). The reader is asked to keep this point in mind as this body of literature is reviewed.

\section{Research on the Association between Bipolarity and Creativity}

In this section the dissention among authors about what can be concluded from research on the association between bipolarity and creativity is discussed. First, a literature review conducted by Waddell (1998) on the relationship between creativity and mental illness in general is reviewed. Second, specific studies on the association between bipolarity and creativity are analyzed and patterns of findings are highlighted. Third, reasons for conceptualizing temperament, character, and the bipolar spectrum of disorders as continua rather than categories are introduced. Fourth, contemporary research on temperament and facets of creativity are critiqued. This facilitates a discussion about hypothesized temperament/character configurations that could be conducive to facets of creativity. 
Some authors argue that the majority of studies that investigate the relationship between creativity and individuals diagnosed with bipolar disorder are plagued by problems in sampling, methodology, presentation of results, and/or conclusions (Andreasen \& Glick, 1988; Andreasen, 2008; Prentky, 2001; Richards \& Kinney, 1990; Rothenberg, 2001; Santosa et al., 2007; Strong et al., 2005; Waddell, 1998). Thus, disagreement persists about whether conclusions about this potential link can be offered (Andreasen, 2008; Benham, 2005; Chavez-Eakle et al., 2006; Frantom \& Sherman, 1999; Ghadirian et al., 2000; Kinney \& Richards, 2007; Makel \& Plucker, 2008; Rothenberg, 2001; Waddell, 1998). Waddell (1998) conducted a literature review of studies on creativity and mental illness to critique the scientific evidence for this association. Her findings will be discussed and then additional studies specific to bipolarity and creativity will be reviewed.

The literature review of Waddell (1998) was comprised of 29 studies. Overall, she identified nine studies that supported an association between mental illness and creativity, 15 studies that negated the relationship, five studies that concluded severe mental illness impaired creativity, and four studies that had unclear findings. Fourteen of these research designs did not employ standardized measures of either mental illness or creativity (Waddell, 1998). The failure of investigators to define creativity and/or mental illness has been a common limitation of early research (Andreasen, 2008; Waddell, 1998).

Only two of the studies reviewed by Waddell (1998) blindly rated participants and six did not incorporate living individuals into their research. The use of retrospective data has been found to yield elevated rates of participant pathology relative to data of living participants. This happens because investigator assessments of retrospective data often have selection biases (Benham, 2005; Waddell, 1998). Waddell concluded that "many authors apparently suggested 
that mental illness and creativity were positively associated based on finding higher than expected rates of mental illness in selected populations in whom creativity was usually not (and mental illness was not always) measured” (p. 169).

Waddell (1998) indicated there was some evidence that milder bipolar disorders may enhance creativity in some individuals. Specifically, she identified a possible relationship between hypomania and creativity but maintained that this did not prove a general association between mental illness and creativity. Waddell also discussed the content of 34 review articles on the purported relationship between mental illness and creativity. She suggested that the expressed enthusiasm for such a link was not supported by the empirical evidence that she reviewed (Waddell, 1998). Andreason (2008) arrived at a similar conclusion when she stated "there appears to be a strong association between creativity and mood disorders. However, the overall literature supporting this association is relatively weak" (p. 254). These findings suggest that the relationship between creativity and affective disorders is in need of further investigation via increased adherence to standards of empirical research.

Jamison et al. (1980) conducted a study that asked 61 participants diagnosed with a mood disorder (i.e., bipolar I disorder, bipolar II disorder, or unipolar depression) about perceived benefits of various experiences associated with their respective disorders. The authors found the majority of participants who had experienced a hypomanic episode connected it to increased sensitivity, sexual intensity, productivity, creativity, and social comfort.

Jamison et al. (1980) acknowledged the limitations of retrospective self-report data but emphasized that subjective attributions of hypomanic episode(s) are important because these lines of thought contribute to whether an individual demonstrates treatment adherence. Another limitation is that although the authors asked participants about their impressions of the 
hypomanic episodes they had experienced, information about how many of their participants were diagnosed with bipolar I or bipolar II disorder was not provided.

Richards and Kinney (1990) assessed the perceptions related to mood and creativity in 48 individuals diagnosed with unipolar depression, bipolar I disorder, or bipolar II disorder (they also included two individuals diagnosed with cyclothymic disorder in this group) via a questionnaire. They found that elevated moods characterized by symptoms of hypomania were viewed by participants as being more conducive to creativity than either manic or depressive episodes. In fact, the more extreme mood swings were perceived to be destructive of creative efforts. Aspects of hypomania that Richards and Kinney hypothesized to be relevant to creativity included changes in "cognition (e.g., rapid thinking), affect (e.g., euphoria), as well as factors influencing motivation (e.g., energy)” (p. 213).

There are several aspects of the Richards and Kinney (1990) study that should be noted when considering their findings. First, the sample was taken from individuals who attended a nationally recognized self help group for major affective disorders. The authors stated the participants in their research "were selected because of their high-functioning characteristics, including the potential for a range of creative experiences, and a very high personal awareness of the nature and features of their illness” (p. 209). Second, Richards and Kinney reported the modal distribution of educational level differed among their participants by diagnostic category (i.e., 18 years of education for those diagnosed with unipolar depression, 17 years of education for those diagnosed with bipolar II disorder, and 16 years of education for those diagnosed with bipolar I disorder). However, they did not assess whether these differences were statistically significant. Collectively, the relatively high functioning level of this sample may limit the 
generality of the findings. Third, the researchers determined diagnoses by relying on the self report of participants subsequent to reviewing diagnostic criteria with these individuals.

It seems that some individuals who experience mild mood elevation (e.g., symptoms of a hypomanic episode) perceive this experience to be conducive to their creative efforts (Andreasen, 2008; Jamison et al., 1980; Richards \& Kinney, 1990). However, there are also people who identify labile moods as impairing their creativity (Andreasen, 2008; Rothenberg, 2001).

Richards et al. (1988) compared the creativity scores of 77 individuals diagnosed with bipolar disorder, cyclothymic disorder, normal first degree relatives of individuals diagnosed with a major affective disorder (e.g., bipolar disorder, unipolar depression, "neurotic depression,” and "affect reaction”), and individuals with no personal or family history of selected types of mental illness (i.e., major affective disorder, cyclothymic disorder, or schizophrenia). The measure of creativity they utilized was the Lifetime Creativity Scales (LCS), which includes seven scales that assess quality and quantity of creative accomplishments. These authors concluded "overall peak creativity may be enhanced, on average, in subjects showing milder and, perhaps, sub-clinical expressions of potential bipolar liability (i.e., those diagnosed with cyclothymic disorder and normal first-degree relatives)” (p. 287). However, the differences between the groups were not statistically significant (Waddell, 1998). Richards et al. (1992) replicated the findings above in two independent studies but they labeled this research "preliminary" due to small sample sizes. Despite the aforementioned limitations of this research, the results suggest that the milder aspects of the bipolar spectrum of disorders (e.g., temperament) may be facilitative of aspects of creativity. 
Frantom and Sherman (1999) assessed the link between family history of affective illness, presence of affective instability, and creativity. They utilized a sample of 54 visual artists who were either undergraduate students or faculty of a fine arts program at an east coast college. These individuals were administered two measures of creativity, the Family History-Research Diagnostic Criteria (FH-RDC), and the General Behavior Inventory (GBI). The creativity measures included the Sensation Seeking Scale (SSS) and the Khatena-Torrance Creative Perception Inventory (KTCPI) (Frantom \& Sherman, 1999).

The SSS is designed to assess a sensation seeking personality style, which is characterized as a need for varied, novel, and complex sensations and experiences to maintain an optimum level of arousal. In addition, those with a sensation seeking disposition are presumed to be more sensitive to inner experiences while conforming less to external constraints. The KTCPI is a personality-biographical inventory that is comprised of two instruments referred to as What Kind of Person Are You? (WKOPAY) and Something About Myself (SAM). The WKOPAY is a measure of creative behavior while the SAM is an autobiographical instrument that identifies creative striving and thinking (Frantom \& Sherman, 1999).

Frantom and Sherman (1999) found that individuals who reported a family history of affective illness were significantly more likely to experience affective instability than those who did not have any such family history. The authors also concluded that there was a strong relationship between creativity and affective instability. In this study, creativity was defined as "self perception, biographical profile, and the willingness to take chances" and affect was defined as a "manifestation of depressive-like and hypomanic-like behavioral fluctuations" (p. 21). Frantom and Sherman emphasized how the creativity measure that accounted for the most variance of affective instability (i.e., SSS) assessed characteristics that they considered to be 
consistent with symptoms of hypomania (i.e., strong need for stimulation, excitement, complex sensations and experiences, and maintenance of an optimum level of arousal). The authors noted that sensation seeking, as measured by the SSS, has been found to be useful in predicting creative behavior. It is also notable that the WKOPAY did not have a significant correlation with affect (Frantom \& Sherman, 1999). It is likely that some qualities of the creativity construct are influenced by affect while others are not. Furthermore, the symptoms relating to hypomania as identified by the authors can also be associated with a hyperthymic temperament.

The proposition that individuals who are diagnosed with milder forms of bipolar disorder (i.e., absence of a manic episode) are typically more creative than those diagnosed with more severe forms of bipolar disorder (i.e., presence of a manic episode) has received some support (Akiskal \& Akiskal, 1988; Frantom \& Sherman, 1999; Goodwin \& Jamison, 1990; Kinney \& Richards, 2007; Richards, 2001; Simonton, 1999; Waddell, 1998). Some authors have purported that individuals diagnosed with cyclothymic disorder may have a creative advantage because their mood states are of moderate intensity relative to those who experience hypomania or mania (Akiskal \& Akiskal, 1988; Akiskal, Savino, et al., 2005; Richards, 2001).

Kinney and Richards (2007) hypothesized that creativity has an “inverted-U” relationship to different levels of mood. They stated "several behavioral characteristics aid creativity when they are moderately elevated, as often occurs with mild mood elevation, but become harmful when the characteristics become too great, as often occurs during the extreme mood elevation of mania.” (p. 229). In addition, Kinney and Richards contend that familial liability for mood disorders may be a third factor that influences the relationship between affective instability and creativity. 
People who have a familial liability for the bipolar spectrum of disorders but are not diagnosed with such a condition tend to be more creative than individuals in the general population. These individuals are also likely to be more creative than their family members who are diagnosed with a bipolar disorder (Jamison et al., 1980; Kinney \& Richards, 2007; Simonton, 1999). This is because they are likely to experience labile emotion but this affective instability does not meet the threshold for a diagnosable condition. In essence, relatives of those diagnosed with a bipolar spectrum disorder are likely to experience the creative benefits of mild affective instability without having to contend with the debilitating influence of extreme moods. This is consistent with the finding that individuals who experience some instability of affect are more likely to be creative than those who have less labile moods (Frantom \& Sherman, 1999; Kinney \& Richards, 2007).

Familial liability for a bipolar spectrum disorder may bestow a creative advantage by exposing these individuals to a specific range of experience that facilitates creative production (Jamison, 1993; Kinney \& Richards, 2007). This is likely to pertain to an over-inclusive cognitive style (ranging from an adaptive level of original thinking to a maladaptive level epitomized by a thought disorder), increased emotional sensitivity (ranging from enhanced appreciation to a maladaptive emotional instability), and inspired motivation (ranging from energetic confidence to maladaptive grandiosity) (Frantom \& Sherman, 1999; Kinney \& Richards, 2007; Richards \& Kinney, 1990; Schuldberg, 2001). All of these qualities are consistent with how a hyperthymic temperament is defined by Akiskal (2004). Moreover, as mentioned above, hyperthymia frequently manifests in individuals who either have a diagnosis of major depressive disorder or have a family history of bipolar disorder. 
Schuldberg (2001) pooled samples from previous research to investigate the possible relationship between six domains of subclinical symptoms of psychopathology and creativity. Assessed subclinical symptoms included positive symptom schizotypal cognitive traits (i.e., positive thought disorder), negative symptom schizotypal affective traits (i.e., flat affect), hypomania, depression, and impulsivity. These symptoms were considered subclinical because each was assessed in isolation in a "non-clinical, non-eminent college student population" rather than being defined as part of any particular diagnostic category (Schuldberg, 2001, p. 11). Schuldberg acknowledged the limitation of "the empirical distinctness or overlap of hypomania, impulsivity, and positive schizotypal traits” (p. 9). The sample consisted of 1,108 participants (Schuldberg, 2001).

Schuldberg (2001) utilized several measures to assess the aforementioned six domains of psychopathology. These tools included the Wisconsin Investigators' Perceptual AberrationMagical Ideation Scale (Per-Mag), Wisconsin Hypomanic Traits scale, Wisconsin Impulsive Nonconformity scale, and the Minnesota Multiphasic Personality Inventory-2 (MMPI-2). The creativity measures included the Barron-Welsh Art Scale (BWAS), How Do You Think (HDYT), Adjective Check List Creative Personality Scale (ACL-CPS), Adjective Check List Domino Scale (ACL-D), and the Peak Vocational and Avocational Creativity ratings of the LCS (Schuldberg, 2001). The BWAS assesses cognitive and affective contributions to creativity because it requires both visual and affective processing. The HDYT is a measure of beliefs, attitudes, traits, and behaviors that sample affective and motivational aspects of creativity. The ACL is a gauge of personality traits that have been linked to creative functioning. ACL-CPS and ACL-D represent different scoring systems of the ACL (Santosa et al., 2007; Schuldberg, 1990). 
Schuldberg (2001) calculated the correlations between the subclinical symptom assessments and the battery of creativity measures. He also conducted several regression models to predict variance in creativity measures with indices of hypomania (i.e., Wisconsin Hypomanic Traits scale or MMPI-2 scale 9), schizotypal symptoms (i.e., Per-Mag scale), impulsivity (Wisconsin Impulsive Nonconformity scale), and the schizotypal negative symptom of flat affect (Wisconsin Physical Anhedonia scale) as independent variables. Schuldberg concluded there was "substantial overlap in the variance that creativity tests shared with psychometric measures of hypomania and subclinical schizophrenic-like positive symptoms. Hypomania was generally the construct accounting for the lion's share of the variance in these creativity test scores” (p. 13). The HDYT, BWAS, ACL-CPS, and ACL-D had significant positive correlations with traits of hypomania. Schuldberg (2001) noted how the psychometrically assessed depressive symptoms were negatively correlated with creativity test scores.

The Schuldberg (2001) study had some noteworthy limitations. First, demographic information (e.g., age, race, level of education, etc.) of the pooled sample was not provided. Second, Schuldberg constructed "several regression models" but only provided information on two of these models (p. 12). One of the stated purposes of the study was to "dispute claims that the overlaps between creativity and psychopathology are best accounted for as a function of the symptoms of affective disorder or of impulsivity alone” (p. 5). However, his research provided more support for a relationship between creativity and traits of hypomania than any other subclinical symptom type (Schuldberg, 2001).

Schuldberg (2001) discussed how his findings represent a curvilinear relationship between hypomanic traits and creativity, which is consistent with the "inverted U" hypothesis proposed by Richards and Kinney (2007). Investigation of this possible nonlinear association is 
founded on two assumptions. First, both mood disorders and creativity are conceptualized as continua rather than discrete categories (Eysenck, 1995; Prentky, 2001; Richards, 2001; Schuldberg, 2001). As such, varying levels of characteristics on a dimension can be assessed across individuals (i.e., shared trait research) and within the same individual over time. Second, mood disorders are thought to be dynamic (i.e., demonstrating a pattern of change over time). Thus, the study of such a curvilinear relationship is the investigation of a nonlinear dynamic system (Schuldberg, 2001). In other words, the relationship between creativity and mood disorders can be viewed "as emerging from a system of interacting components, changing with time, and with nonlinear causal connections linking at least some of the contributing variables” (Schuldberg, 2001, p. 14).

The debate about whether mental disorders should be viewed as discrete conditions or arbitrary distinctions on a continuum is longstanding. However, limitations of the categorical model are becoming increasingly recognized (e.g., no demonstrated boundaries between diagnostic categories, high comorbidities between disorders, high degree of short term diagnostic instability, etc.) (Eysenck, 1995; Widiger \& Mullins-Sweatt, 2007). On the other hand, categorical and dimensional views of psychopathology do not necessarily have to be mutually exclusive. Cloninger et al. (1994) discussed how work with the TCI has demonstrated that a dimensional approach can be helpful in delineating underlying determinants of personality (i.e., specific dimensions of temperament and character) while a categorical approach can be of use in describing groups of individuals who embody unique patterns of interactions between the dimensions (i.e., temperament and character configurations).

The reader will recall that the psychobiological model of temperament and character as well as the bipolar spectrum of disorders are conceptualized as dimensional. Moreover, the 
psychobiological model of temperament is based on empirical observations that personality development behaves as a nonlinear adaptive system. In fact, such findings establish the "need for psychobiologists to think of personality development as a self-organizing multidimensional process rather than to continue to use linear models of quantitative traits or categories” (Cloninger et al., 1997, p. 890). Empirical research that investigates the relationship between temperament and facets of creativity will now be reviewed.

Akiskal, Savino et al. (2005) conducted an exploratory study to generate preliminary data on temperament profiles of psychiatric outpatients. The sample constituted a record review of patients in the private practices of each author. The participants were divided into groups based on occupation, which included lawyers (34), physicians of different specialties (41), architects (27), a mixed group of artists (48), journalists (34), managers/executives (35), and industrialists (48). Akiskal, Savino, et al. compared these groups to a control group. These professional groups and the control group were matched for age, gender, and social class. Furthermore, the groups were paired according to mental illness with respect to broad diagnostic categories (e.g., bipolar disorder, unipolar depression, anxiety). The temperaments of these individuals were assessed using criteria that were validated as part of the Temperament Evaluation of Memphis, Pisa, Paris, and Sand Diego-Interview Version (TEMPS-I) (Akiskal, Savino et al., 2005).

Akiskal, Savino et al. (2005) found that each professional group had a distinct temperamental style. In comparison to the control group, the group of mixed artists was four times as likely to demonstrate a cyclothymic temperament. However, Akiskal, Savino et al. also described their analyses as exploratory and acknowledged how their results must be considered tentative due to the research design they employed. For example, Akiskal, Savino et al. indicated they could not entirely rule out the possibility that the temperamental profiles they identified 
were characteristic of their participants' mental illnesses. On the other hand, they maintained that "the rather distinct profiles that emerged for each of the seven professional domains argues against the notion that the underlying psychiatric illnesses fashioned the rates of temperament in each of the professions” (p. 204). Additional noteworthy limitations include the utilization of mixed groups of some professions (i.e., artists, physicians) and that the categorization of participants into professional groups was not always straightforward (Akiskal, Savino et al., 2005).

A series of four articles from a research group at Stanford University (i.e., Nowakowska et al., 2005; Santosa et al., 2007; Srivastava et al., 2010; Strong et al., 2007) analyzed different aspects of data collected from the same group of participants. The sample will first be described and then the contributions of each article will be discussed in turn. Finally, the limitations of these studies will be delineated.

Four instruments of creativity were utilized to assess non-eminent creativity in the participants. The creativity measures included the BWAS, ACL-CPS, and the Torrance Tests of Creative Thinking - Figural (TTCT-F) and Verbal (TTCT-V) forms (Nowakowska et al., 2005; Santosa et al., 2007; Srivastava et al., 2010; Strong et al., 2007). Santosa et al. (2007) calculated correlations between each of these creativity measures and the only significant association was between the TTCT-V and TTCT-F. They suggested that "this is consistent with measures reflecting independent facets of creativity or independent constructs separately related to creativity” (p. 35). Schuldberg (1990) also found an insignificant correlation between the BWAS and ACL-CPS.

The participants were also administered three self-report measures of temperament. These instruments included the Temperament Evaluation of the Memphis, Pisa, Paris, and San 
Diego Autoquestionnaire (TEMPS-A), Temperament and Character Inventory (TCI), and Revised NEO Personality Inventory (NEO PI-R). The TEMPS-A assesses four affective dimensions of temperament that include Cyclothymia, Hyperthymia, Dysthymia, and Irritability. The NEO PI-R is a measure of the five aspects of personality that are referred to as Neuroticism, Extraversion, Openness, Agreeableness, and Conscientiousness (Nowakowska et al., 2005; Santosa et al., 2007; Srivastava et al., 2010; Strong et al., 2007).

The sample consisted of 153 participants that were divided into the following groups; those diagnosed with one of three bipolar disorder diagnoses (i.e., bipolar I disorder, bipolar II disorder, and bipolar disorder not otherwise specified), participants diagnosed with major depressive disorder, creative controls (i.e., individuals recruited from graduate programs in Creative Writing, Fine Arts, and Product Design at Stanford University), and healthy controls. All participants underwent a psychiatric evaluation that included a detailed medical and psychiatric evaluation for DSM-IV diagnoses as assessed by the Structured Clinical Interview (SCID). The people in the healthy control group (HC) had no personal or first degree relative history of a psychiatric disorder, substance abuse, or neurological illness. Those in the creative control group (CC) had no current psychiatric or substance abuse problems, but the majority of these individuals met SCID criteria for a past psychiatric disorder. This is an important distinction of the CC group and it will be revisited when limitations are reviewed below. The number of people in each group included 49 BP, 25 MDD, 32 CC, and 47 HC (Nowakowska et al., 2005; Santosa et al., 2007; Srivastava et al., 2010; Strong et al., 2007).

The participants in the creative control group (CC) engaged in a range of artistic mediums that included studio artists (i.e., painting, photography, sculpture, or multimedia), creative writers (i.e., fiction, poetry), and product designers (i.e., Master of Fine Art or Master of 
Science in Mechanical Engineering). All of the participants had at least two years of education and the average educational level was a four year college degree. Participants in the CC group had a significantly higher level of education than the other groups (Nowakowska et al., 2005; Santosa et al., 2007; Srivastava et al., 2010; Strong et al., 2007).

Exclusion criteria for individuals who were diagnosed with major depressive disorder (MDD) or a bipolar disorder (BP) included primary diagnoses other than MDD or BP, active substance abuse, personality, or eating disorders. Seventy five percent of individuals diagnosed with BP were taking medication at the time of data collection (Nowakowska et al., 2005; Santosa et al., 2007; Srivastava et al., 2010; Strong et al., 2007).

Nowakowska et al. (2005) utilized the data from the aforementioned sample to investigate the temperamental similarities and differences between participant groups. These authors found that those in the creative control group (CC) were more similar to individuals diagnosed with a mood disorder than people in the healthy control group (HC). Specifically, in comparison to people in the HC, the other groups (i.e., BP, MDD, and CC) evidenced higher incidence of Cyclothymia, Dysthymia, Irritability, Neuroticism, Harm Avoidance, and Novelty Seeking. On the other hand, those in HC demonstrated higher levels of Conscientiousness and Self-Directedness (Nowakowska et al., 2005). Nowakowska et al. also found differences between the people in CC, BP, and MDD despite the substantial temperamental commonalities between these groups. Specifically, participants in BP had Cyclothymia scores that were almost $50 \%$ higher than those of MDD or HC. Individuals in the CC and BP also were differentiated from people in HC by their higher Openness scores (Nowakowska et al., 2005).

Nowakowska et al. (2005) concluded that participants in creative control group (CC) and the bipolar diagnoses group (BP) had score profiles that contained more overlap than either 
group had with those diagnosed with major depressive disorder (MDD). When compared to people in MDD, individuals in CC/BP were more similar in terms of Cyclothymia, Irritability, Dysthymia, and Neuroticism. The authors purported that collectively, these commonalities could represent the shared aspects of temperament that contribute to the elevated creativity in the BP and CC groups. This point will be revisited when the findings of Strong et al. (2007) are reviewed below.

Santosa et al. (2007) relied on the aforementioned data to assess the purported relationship between individuals diagnosed with a bipolar disorder and elevated non-eminent creativity. They found that participants in BP/CC performed significantly better than those in the healthy control group (HC) in terms of BWAS scores. On the other hand, individuals in BP and HC demonstrated similar scores on the ACL-CPS, TTCT-V, and TTCT-F. Santosa et al. (2007) concluded that their findings regarding the BWAS scores could be related to temperamental/personality differences among people in BP/CC, MDD, and HC (Nowakowska et al., 2005).

Strong et al. (2007) explored the relationships between temperament and creativity among the different groups of the aforementioned data set. These authors conducted a factor analysis of the temperament measures and identified a Neuroticism/Cyclothymia/Dysthymia Factor that accounted for some of the group differences on the BWAS as identified by Santosa et al. (2007). This factor was primarily composed of two scales from the TEMPS-A (i.e., Cyclothymia, Dysthymia) and one scale from the NEO PI-R (i.e., Neuroticism). Moreover, Strong et al. suggested that the BWAS is a measure of the facet of creativity that is enhanced by these temperamental attributes. 
Strong et al. (2007) stated "it may be that Neuroticism/Cyclothymia/Dysthymia provides a creative advantage by increasing access to a range of affective experience, particularly negative affect (Neuroticism) and changeability of affect (Cyclothymia)" (p. 45). Therefore, participants in the creative control and bipolar diagnoses groups (CC/BP) may be more creative than people in the other groups in terms of the facet of the construct assessed by the BWAS. This is likely due to the ability of these individuals to experience intense and varied affect relative to those who do not necessary have such an affective range (i.e., those diagnosed with major depressive disorder or the health control group). Strong et al. confirmed these differences by performing exploratory analyses of the individual correlations between BWAS scores and the items that comprised the Neuroticism/Cyclothymia/Dysthymia Factor. The authors concluded that "direct relationships existed between BWAS-Total scores and NEO Neuroticism and TEMPS-A Cyclothymia that were at least to some degree independent from one another” (p. 46).

Srivastava et al. (2010) analyzed a subset of the aforementioned data. Specifically, 117 of the original 153 participants were administered the Myers-Briggs Type Indicator Form M (MBTI) in addition to the other measures that were previously mentioned. The number of individuals in each subgroup included 32 with a bipolar diagnosis (BP), 21 diagnosed with major depressive disorder (MDD), 22 in the creative control group (CC), and 42 in the healthy control group (HC). The relationships between the subgroups, temperament/personality measures, and creativity measures reported by the other three studies remained (e.g., the differences between people in BP/CC and the other subgroups were consistent with the previously reported findings when the MBTI data were considered). The purpose of this study was to extend the findings of the other studies by investigating the possible relationship between facets of creativity, individuals diagnosed with a mood disorder, and cognitive style. Srivastava et al. hypothesized 
"that creativity in patients with bipolar disorder may be related not only to negative and changeable feelings but also to intuitive thinking” (p. 2).

Srivastava et al. (2010) offered two major conclusions. First, it is likely that there is an affective temperamental component of creativity that is assessed by the BWAS. Second, there may also be a cognitive temperamental component of creativity assessed by the BWAS.

Srivastava et al. also suggested that the relationship between affective temperament and the BWAS may be more robust than the relation between cognitive temperament and the BWAS. They suggested that more research should be conducted on both aspects of temperament and the facet of creativity assessed by the BWAS. The questionable psychometric properties of the MBTI are a limitation specific to this study (Srivastava et al., 2010).

There are several noteworthy limitations of the four studies reviewed above (i.e., Nowakowska et al., 2005; Santosa et al., 2007; Srivastava et al., 2010; Strong et al., 2007). First, the sample was comprised of both individuals who were taking medication and some who were not. A second limitation was the small size of the sample subgroups, which limits the statistical power of the studies. Santosa et al. (2007) stated that "statistical power limitations could account for some of our negative findings, and particularly limit our ability to assess creativity in the subgroups of the CC and BP groups” (p. 37). Along these lines, although the CC and BP groups differed in terms of state symptoms (i.e., members of the CC group did not qualify for SCID criteria for a psychiatric disorder at the time of the study), these groups may have been similar in regard to trait symptoms (i.e., many of the individuals in the CC group met SCID criteria for a past psychiatric disorder). These commonalities could account for the temperament profiles shared by these two groups. Third, the high educational level of the sample limits the generalizability of the findings. Fourth, the creative control group consisted of students rather 
than artists in their respective fields. In addition, these participants were from several different artistic mediums so differences between divergent approaches could not be assessed (Nowakowska et al., 2005; Santosa et al., 2007; Srivastava et al., 2010; Strong et al., 2007).

Neuroticism is a well defined construct of traditional personality theory but it may not be specific enough to discriminate between temperamental differences of individuals diagnosed with major depressive disorder, the bipolar spectrum of disorders, and those with no diagnosable mood disorder (Strong et al., 2005). Akiskal et al. (2006) found that individuals diagnosed with bipolar disorder I scored low on neuroticism as assessed by a variety of self-rated personality scales whereas those diagnosed with bipolar disorder II and major depressive disorder scored high on this construct. In addition, the items that underpinned neuroticism for bipolar disorder II and major depressive disorder participants differed. Items associated with neuroticism for those diagnosed with bipolar disorder II were mostly due to symptoms of cyclothymia while people diagnosed with major depressive disorder were more likely to endorse items consistent with subthreshold depressive traits (Akiskal et al., 2006). Moreover, the TEMPS-A subscale of Cyclothymia was the only measure of temperament/personality that differentiated participants in BP from those in MDD in the Nowakowska et al. (2005) study.

Utilizing neuroticism to distinguish people who experience different aspects of the bipolar spectrum is problematic because the construct is unable to capture subtle differences between individuals whose experiences are at different points of the affective continuum. This would constitute the same error that earlier researchers made in assessing the relationship between bipolarity and creativity without attending to nuanced demarcations of affect (i.e., the soft bipolar spectrum). 
It may be that another subset of features (e.g., sense of well-being, confidence, intense feelings, and enthusiasm) may underpin creativity in individuals who are optimally creative (Richards, 2001; Richards \& Kinney, 1990). In other words, the creative advantages associated with the milder forms of bipolar disorder could be due to psychological variables other than the illness itself. These personality variables may include ability to generate numerous ideas (i.e., creative thinking), receptivity to novel ideas and experiences, increased level of confidence, perseverance, and continuous striving for achievement. Each of these variables has similarities with the symptoms of a hypomanic or manic mood state but can become detrimental at the intensity associated with these respective diagnostic conditions (Eysenck, 1995; Kinney \& Richards, 2007; Richards, 2001; Simonton, 2002; Strong et al., 2005). Thus, as discussed earlier, a hyperthymic temperament may best account for previous findings relating to bipolarity and optimal creativity.

Chavez-Eakle et al. (2006) conducted a study to explore the relationship between creativity, temperament/character, and psychopathology. They administered the TCI, TTCT Figural and Verbal forms, and the Symptom Check List (SCL-90) to 90 participants from Mexico City who were divided into three groups. Group I (GI) consisted of 30 individuals who were members of the National System of Researchers or the National System of Creators in Mexico. These organizations provide support to the most prolific scientists and artists in Mexico and members are required to maintain continuous and successful achievement in their respective fields. Group II (GII) was the control group and consisted of 30 people who were administrative staff or graduate students of a university in Mexico, employees of a Mexico institute of psychiatry, and members of a parents association. These participants all underwent a clinical 
interview based on the DSM-IV and did not endorse any symptoms of a psychiatric disorder (Chavez-Eakle et al., 2006).

Group III (GIII) constituted 30 individuals who were psychiatric outpatients at a Mexico institute of psychiatry and the diagnosis of these participants was confirmed by three psychiatrists. Exclusionary criteria for GIII included acute psychosis, dementia, or psychopharmacological treatment. In describing the various diagnoses of people in GIII, ChavezEakle et al. (2006) stated “the included diagnoses were mainly major depressive disorder and anxiety disorders (panic attacks, general anxiety), but other diagnoses included social phobia, obsessive-compulsive disorder, and bipolar disorder.” (p. 30). There were no significant differences in age or gender among the three groups (Chavez-Eakle et al., 2006).

Chavez-Eakle et al. (2006) found that the three groups significantly differed on the composite score of the TTCT with GI performing the highest and GIII scoring the lowest. This trend was also evident for all of the creative aspects assessed by the TTCT except for fluency (i.e., flexibility, originality, elaboration, resistance to premature closure, abstractness of titles). Moreover, the authors identified a temperament and character profile that was associated with high scores on the TTCT and high creative achievement. Participants in GI scored significantly higher than the other groups on exploratory excitability (a facet of the Novelty Seeking temperament scale), Self-Directedness (a character scale), and Cooperativeness (a character scale) while scoring significantly lower on Harm Avoidance (a temperament scale). In addition, people in GI evidenced more Persistence (a temperament scale) than GII or GIII individuals, but the difference in scores was not significant (Chavez-Eakle et al., 2006).

Chavez-Eakle et al. (2006) elaborated on the temperament and character profile that emerged from their sample of creative individuals. First, the authors suggested that elevated 
exploratory excitability scores indicated that these participants are frequently explorative in new situations. Second, high Self-Directedness scores demonstrated that they are often responsible, goal directed, resourceful, self accepting, and congruent. Third, elevated Cooperativeness revealed that these people are likely empathic and tolerant of others. However, they are probably autonomous and self determining. Fourth, their low Harm Avoidance scores indicated that they are usually optimistic, embrace uncertainty, and are typically energetic (Chavez-Eakle et al., 2006).

There were some limitations of the Chavez-Eakle et al. (2006) study. First, GII consisted of a range of individuals from different backgrounds (i.e., graduate students, administrative staff, and employees of a psychiatric institute) and a formal assessment (e.g., SCL-90) of psychological disorders was not conducted prior to creation of the group. Second, the authors did not provide details regarding the diagnoses of GIII participants other than what was conveyed in the quote above. Chavez-Eakle et al. did control for differences in education among the groups and reported that education did not influence their results. Finally, it has been suggested that the TTCT measures aspects of creativity that are not related to temperament/personality (Strong et al., 2007). Chavez-Eakle et al. acknowledged they did not consider psychopathology as an exclusionary criterion for membership in GI. However, they reported no significant differences in psychopathology between GI and GII as assessed by the SCL-90. In addition, individuals in both GI and GII had significantly lower psychopathology scores than GIII.

A variety of personality traits have been associated with creativity including adventurousness, nonconformity, assertiveness, introspection, self criticalness, emotional sensitivity, psychoticism (i.e., defined as a latent trait underlying a variety of psychotic disorders and is characterized by impulsivity, hostility, and aggression), originality, independence, 
increased risk taking, high personal energy, curiosity, humor, affinity for the complex and novel, openness, private, tolerance for ambiguity, confidence, arrogance, persistence, impulsivity, resilience, introversion, autonomy, achievement oriented, imaginative, hostile, need for power, unconventional, cognitive/behavioral flexibility, ambitious, aloof, maintaining broad interests, and accommodating of conflicting traits in one’s self-concept (Akiskal, Savino, \& Akiskal, 2005; Baer \& Kaufman, 2006; Barron \& Harrington, 1981; Caselli, 2009; Eysenck, 1995; Feldhusen \& Goh, 1995; Makel \& Plucker, 2008; Mumford, 2003; Schuldberg, 2001; Simonton, 2002). It is important to note that many of these attributes exhibit strong correlations with heritability (Simonton, 2002).

Feist (1999) conducted an extensive review of the literature on the relationship between creativity and personality traits, which is frequently cited by other authors (e.g., Baer \& Kaufman, 2006; Galang, 2010; Mumford, 2003; Simonton, 2002; Qian, Plucker, \& Shen, 2010). This review was the first to summarize research findings that distinguish personality traits associated with the creative scientist and artist. For the purposes of the review, the term "artist" was defined as including visual artists (i.e., painters, sculptors, cinematographers, photographers, architects), literary artists (i.e., writers, poets), and performing artists (i.e., musicians, singers, dancers, actors). In a similar vein, studies comparing more and less creative scientists were included since the field of science is comprised of different vocations with differing needs for creativity. The science category included individuals in natural science, biological science, social science, engineering, invention, or math (Feist, 1999).

Feist (1999) concluded that artists are likely to be imaginative, open to new ideas, neurotic, affectively labile, asocial, and even antisocial at times. Likewise, he proposed that the creative scientists could best be described as open, flexible, ambitious, asocial, arrogant, and 
hostile. Therefore, “creative people in art and science do not share the same unique personality profiles: Artists are more affective, emotionally unstable, as well as less socialized and accepting of group norms, whereas scientists are more conscientious” (Feist, 1999, p. 290). It is noteworthy that Feist did not provide a critique of the studies he reviewed and his findings included some research that was criticized by Waddell (1998) (e.g., Andreasen \& Glick, 1988; Jamison, 1993; Ludwig, 1995).

It is difficult to establish a consistent and comprehensive list of creative personality traits from the vast literature that exists because many of the identified traits are conflicting (Galang, 2010; Qian et al., 2010). This point is elegantly captured by Galang (2010) when he stated that creative individuals "are thought to consist of equal parts of confidence and fragility, arrogance and sensitivity. They are unconstrained and focused, found to be terrifying and at other times inspiring” (p. 1241).

It is likely that creativity is the end result of the interplay between a variety of factors and it probably manifests from more than one combination of these variables (Caselli, 2009; Feist \& Barron, 2003; Galang, 2010; Richards, 2001). This may explain why different traits are associated with the artists versus the creative scientists. Feist and Barron (2003) suggested that traits associated with creativity may not act as causal agents but rather serve to lower behavioral thresholds conducive to creativity. In this functional threshold view, there is an interaction between traits and situations such that particular dispositions increase the number of situations in which a given trait congruent behavior may be expressed. Therefore, the possession of a combination of creative traits effectively lowers an individual's threshold for creative behavior (Feist \& Barron, 2003). 
Caselli (2009) provided an organizational schema of creativity that is consistent with the functional threshold view proposed by Feist and Barron (2003). He identified five possible interactive components that purportedly interact in the genesis of creativity. These components include motivation, perception, action, temperament, and social interaction. The creative individual must be able to perceive a need for some change in an area. She also possesses motivation to create a plan of action to enact her vision and be able to interact with others in a way that conveys the importance of her creation to society. In addition, the creative individual must have a temperament that enables successful navigation of these processes (Caselli, 2009). Although Caselli utilized the psychobiological model of temperament and character to operationalize the construct of temperament, he does not identify a specific temperament configuration that could be likely to facilitate creativity.

Cloninger (2004) identified possible temperament and character configurations that are hypothetically associated with creativity. Specifically, he suggested that individuals who are high on all three character dimensions (i.e., Self-Directedness, Cooperativeness, and SelfTranscendence) are likely to be creative. Interestingly, he distinguished this character configuration from individuals who have a cyclothymic character configuration (i.e., low on Self-Directedness but high on the other two character dimensions) that is referred to as "Moody." In essence, the two configurations differ only on Self-Directedness (Cloninger, 2004).

Hyperthymic, depressive, and cyclothymic temperaments, as defined by Akiskal and his colleagues, have been found to have distinct configurations on the TCI. Within the terminology of the psychobiological model of temperament and character, a hyperthymic temperament is referred to as a "Passionate” temperament and consists of high Novelty Seeking, high Reward Dependence, and low Harm Avoidance (the reader will recall that Persistence is not included in 
temperament configurations). A cyclothymic temperament is referred to as a "Reliable" temperament and is comprised of low Novelty Seeking, high Reward Dependence, and low Harm Avoidance. Thus, the "Passionate” (i.e., hyperthymic) and "Reliable” (i.e., cyclothymic) temperaments only differ on the dimension of Novelty Seeking. In contrast, a depressive temperament is referred to as an "Explosive" temperament and is comprised of high Novelty Seeking, low Reward Dependence, and high Harm Avoidance (Cloninger, 2004; Cloninger et al., 1994).

The "Passionate" and "Reliable" temperament configurations were found to be most commonly associated with the "Creative" character configuration in a sample of 593 individuals from the general population. This is an example of the aforementioned principle of equifinality (i.e., different temperament configurations give rise to the same character configuration). However, the "Passionate" temperament has also been frequently found to lead to a "Moody" character configuration whereas the "Reliable" temperament has been commonly associated with an "Organized” character configuration (i.e., high Self-Directedness, high Cooperativeness, and low Self-Transcendence). This is an example of the aforementioned principle of multifinality (i.e., the same temperament configuration leads to different character configurations) (Cloninger, 2004; Cloninger et al., 1997).

Chavez-Eakle et al. (2006) indicated they were the first to investigate whether these putative creative configurations actually manifest in a sample of creative individuals. To date, no further research has been found that addresses the subject.

\section{Conclusions}

Biographical methods and shared-trait research are two ways in which the relationship between creativity and mental illness has been investigated. Retrospective studies of biographical 
information have many limitations (Andreasen, 2008; Benham, 2005; Prentky, 2001; Richards \& Kinney, 1990; Rothenberg, 2001; Santosa et al., 2007; Schuldberg, 2001; Waddell, 1998). In shared-trait research, psychometric assessment is conducted to identify characteristics that are associated with creativity (Benham, 2005; Schuldberg, 2001). One benefit of this approach is that it can highlight the shared and unshared elements of creativity and psychopathology. This allows for the possibility of a relationship between creativity and healthy traits that may serve to mitigate the impact of coexisting problematic symptoms. A drawback of past shared-trait research is that it has largely been conducted in the absence of a guiding theory that accounts for how various characteristics are linked to creativity (Schuldberg, 2001).

The psychobiological model of temperament and character provides a theoretical framework from which to consider the possible relationship between traits and aspects of creativity. Furthermore, the Temperament and Character Inventory - Revised (TCI-R) assesses traits associated with pathology as well as qualities linked to life satisfaction (e.g., virtue, positive emotion) (Cloninger, 2004; Cloninger, 2008). This allows for the simultaneous investigation of the relationship between facets of creativity and positive as well as maladaptive characteristics. The use of a creativity measure is enhanced by an explanation of how it meets a definition of creativity identified by the researcher (Plucker et al., 2004). In addition, this literature review has revealed that the choice of creativity measure is important as it influences what type of relationship (or if a relationship exists) between bipolarity and creativity.

The Barron-Welsh Art Scale (BWAS) will be utilized in this study for two reasons. First, the BWAS demonstrated a relationship with hypomanic traits in the Schuldberg (2001) study as well as to temperament in the research conducted by the group at Stanford University (i.e., Nowakowska et al., 2005; Santosa et al., 2007; Srivastava et al., 2010; Strong et al., 2007). 
Second, it has been found to measure a facet of creativity that is distinct from aspects of the construct assessed by other commonly used measures (i.e., ACLS-CPS, TTCT). The psychometric properties of the BWAS and what it measures will be reviewed in the Methods section. However, it is important to emphasize that in this study, creativity is defined by the BWAS, which assesses some (but not all) aspects of the multifaceted construct. As such, from this point on, when the term creativity is utilized, it indicates facets of creativity as measured by the BWAS.

The bipolar disorders can be viewed as a spectrum of disorders. Some of the major demarcations of this continuum include a major depressive episode, dysthymia, euthymia, hyperthymia, hypomania, and mania (Akiskal \& Akiskal, 1988; Stahl, 2008). Dysthymia, hyperthymia, and cyclothymic disorder represent distinct temperament types within this framework. A hyperthymic temperament is not considered pathological within this framework (Akiskal, 2004; Akiskal, Akiskal, Haykal et al., 2005; Akiskal, Mendlowicz, et al., 2005; Stahl, 2008). There is need for research that identifies how different groups of temperamental traits are related to aspects of creativity (Chavez-Eakle et al., 2006; Mumford, 2003; Nowakoska et al., 2005). Past research on the putative association between bipolarity and creativity is flawed to the extent that it assesses some aspects of the bipolar spectrum of disorders (e.g., BPD I, BPD II) but excludes more subtle parts of the bipolarity continuum (e.g., sub-syndromal symptoms of hypomania, hyperthymic temperament).

The research group at Stanford University found that individuals in a bipolar group (BP) and a creative control group (CC) shared a similar temperamental profile that distinguished them from people in healthy control (HC) and major depressive disorder (MDD) groups. When compared to MDD, CC and BP were more similar in terms of Cyclothymia, Irritability, 
Dysthymia, and Neuroticism (Nowakowska et al., 2005, Santosa et al., 2007; Strong et al., 2007). Nowakoska et al. (2005) suggested that collectively, these commonalities may represent the shared aspects of temperament that contribute to the elevated creativity in the BP and CC groups.

Santosa et al. (2007) found people in the BP/CC groups performed significantly better than those in the HC in terms of the BWAS scores. On the other hand, participants in the BP/CC and HC groups demonstrated similar scores on the ACL-CPS, TTCT-V, and TTCT-F. Santosa et al. concluded that their results regarding the BWAS scores could be related to the aforementioned temperamental/personality differences among BP/CC, MDD, and HC. Therefore, CC/BP people may be more creative than the other groups in terms of the facets of the construct assessed by the BWAS. This is likely due to the capacity of these individuals to experience intense and varied affect relative to those who do not necessarily have such an affective range (i.e., HC, MDD) (Strong et al., 2007). It is important that in this group of studies, the commonalities between the CC and BP groups could be due to how they were not differentiated in terms of possible trait symptoms (i.e., many of the participants in the CC group met SCID criteria for a past psychiatric disorder).

Kinney and Richards (2007) hypothesized that creativity has an "inverted-U” relationship with different levels of mood elevation. This hypothesis is based on the finding that individuals who have a familial liability for the bipolar spectrum of disorders, but are not diagnosed with such a condition, tend to be more creative than people in the general population (Kinney \& Richards, 2007; Richards et al., 1992). These people also tend to be more creative than their family members who are diagnosed with a bipolar disorder since extreme moods can serve to impede creative efforts (Jamison et al., 1980; Richards \& Kinney, 1990; Simonton, 1999). They 
likely experience mood swings but their affective instability does not reach the threshold for a diagnosable condition (Frantom \& Sherman, 1999; Kinney \& Richards, 2007; Richards et al., 1988; Richards et al., 1992).

Familial liability for a bipolar spectrum disorder may bestow a creative advantage by exposing these individuals to a specific range of experience that is most conducive to creative production (Jamison, 1993; Kinney \& Richards, 2007). This benefit likely pertains to an overinclusive cognitive style (ranging from an adaptive level of original thinking to a maladaptive level epitomized by a thought disorder), increased emotional sensitivity (ranging from enhanced appreciation to maladaptive emotional instability), and inspired motivation (ranging from energetic confidence to maladaptive grandiosity) (Frantom \& Sherman, 1999; Kinney \& Richards, 2007; Richards \& Kinney, 1990; Schuldberg, 2001). All of these qualities are consistent with how a hyperthymic temperament is defined by Akiskal (2004).

Investigation of a potential nonlinear association between creativity and mood is based on two assumptions. First, both bipolar disorders and creativity are conceptualized as continua rather than as categories (Eysenck, 1995; Prentky, 2001; Richards, 2001; Schuldberg, 2001). Thus, varying levels of characteristics on a dimension can be assessed across individuals (i.e., shared trait research) and within the same individual over time. Second, mood disorders are thought to be dynamic (i.e., demonstrating a pattern of change over time). The study of such a curvilinear relationship is the exploration of a nonlinear dynamic system (Schuldberg, 2001). In other words, the relationship between creativity and the bipolar spectrum can be viewed "as emerging from a system of interacting components, changing with time, and with nonlinear causal connections linking at least some of the contributing variables” (Schuldberg, 2001, p. 14). 
Categorical and dimensional views of psychopathology do not necessarily have to be mutually exclusive. Cloninger et al. (1994) discussed how a dimensional approach can be helpful in delineating underlying determinants of personality (i.e., the independent dimensions of temperament and character) while a categorical approach can be of use in describing groups of individuals who embody unique patterns of interactions between the dimensions (i.e., temperament and character configurations). The TCI-R is derived from the psychobiological model of temperament and character, which is a model based on empirical observations that suggest personality develops from a nonlinear adaptive system (Cloninger et al., 1997).

Cloninger (2004) identified possible temperament and character configurations that are hypothetically associated with creativity. He suggested that individuals who are high on all three character dimensions (i.e., Self-Directedness, Cooperativeness, and Self-Transcendence) are likely to be creative. This configuration differs from a cyclothymic character configuration (i.e., "Moody") on the dimension of Self-Directedness. In addition, the "Creative” character configuration has been most associated with two temperament configurations (i.e., "Passionate" and "Reliable") in previous research. These configurations only differ on the dimension of Novelty Seeking. Within the bipolar spectrum nomenclature, a "Passionate" temperament configuration equates to a hyperthymic temperament while the "Reliable" temperament configuration corresponds to a cyclothymic temperament. Finally, the "Passionate" and "Reliable" temperament configurations also frequently lead to alternative character configurations (i.e., "Moody” and “Organized,” respectively). These examples of equifinality and multifinality are indicative of a nonlinear (i.e., lack of a one-to-one) relationship between temperament and character (Cloninger, 2004; Cloninger et al., 1994; Cloninger et al., 1997). 
The creative advantages associated with the milder forms of bipolar disorder could be due to distinct forms of temperament. Two of these temperament types could be the aforementioned "Passionate” and "Reliable” temperament configurations. As discussed, either temperament configuration can lead to a "Creative" character configuration, which research has found to be associated with psychological strengths (i.e., positive emotions, satisfying quality of life, and virtuous conduct) rather than labile affect (Cloninger, 2004; Cloninger, 2008; Cloninger et al., 1994).

The possibility of temperament underpinning the creative advantages that have been associated with the bipolar spectrum of disorders could account for two major findings in the literature. First, since temperament has a strong heritable component, it could explain why family members of people diagnosed with a bipolar spectrum disorder have been found to be more creative than either the diagnosed individual or the general population. In essence, it could be that these family members share a common temperament configuration (i.e., "Passionate" or "Reliable”) with the proband but develop a different character configuration. Specifically, they may develop a "Creative” character configuration while their relative(s), who are diagnosed with a bipolar spectrum disorder, may develop a Moody” character configuration. Second, the different combinations of temperament and character configurations may explain the various and conflicting character traits that have been linked to the creative personality. For example, an individual with a "Passionate/Creative" temperament/character configuration could be more likely to become an artist whereas a person with a "Reliable/Creative” temperament/character configuration may be more inclined to become a creative scientist.

The above paragraph is speculative at this point. It is also important to remain aware that creativity probably emerges from several interactive components (Caselli, 2009; Plucker et al., 
2004). For example, it could result from the interplay between motivation, perception, action, temperament, and social interaction (Caselli, 2009). Thus, temperament is likely just one component of the creativity matrix. It is possible that specific temperaments are not causal agents per se but instead serve to lower behavioral thresholds, which increases the likelihood of an individual engaging in a creative act (Feist \& Barron, 2003). Whether creativity actually manifests probably depends on additional parts of the creativity matrix (e.g., the environment). However, the possible relationship between specific temperaments and creativity can potentially contribute to the ongoing debate about the relationship between the bipolar spectrum of disorders and creativity.

Chavez-Eakle et al. (2006) indicated they were the first to investigate whether the putative creative configurations actually manifest in a sample of creative individuals. Their group of 30 individuals from the National System of Researchers and the National System of Creators in Mexico (GI) did score significantly higher on several of the TCI dimensions than either the control or psychiatric groups. The GI scores were a partial match for the "Passionate" temperament. These individuals evidenced low Harm Avoidance and high Reward Dependence. However, their scores on Reward Dependence were not significantly higher than the other groups. In addition, the GI scores were only significantly higher than the other groups on one facet of Novelty Seeking (i.e., exploratory excitability) rather than on the entire dimension. The reader will recall that a high Novelty Seeking score differentiates the "Passionate" from the "Reliable” temperament configuration.

The GI scores were also a partial match for the "Creative” character configuration outlined by Cloninger (2004). While they scored significantly higher than the other groups on Self-Directedness and Cooperativeness, they did not score significantly higher than the other 
groups on Self Transcendence (although this dimension was high as would be predicted by the “Creative” configuration) (Chavez-Eakle et al., 2006).

The "Creative" character configuration and at least one of the temperament configurations most associated with it (i.e., "Passionate" and "Reliable") still needs to be established in a sample of creative individuals. This may be more likely to occur if a sample is divided into homogenous groups based on how they score on a creativity measure (e.g., low, medium, high scorers) and then each group is compared in terms of their temperament and character configurations. Moreover, the BWAS is possibly a better assessment of creativity to use in this case because it has been suggested that the test employed by Chavez-Eakle et al. (2006) (i.e., Torrance Tests of Creative Thinking) measures aspects of creativity that are not related to temperament/character/personality (Strong et al., 2007). Therefore, the purpose of this study is to replicate and extend the findings of Chaves-Eakle et al. (2006). 


\section{CHAPTER 3: Method}

\section{Participants}

I collaborated with the West Virginia University (WVU) College of Creative Arts (CAC) to acquire participants. This has been done by other researchers in university settings (Frantom \& Sherman, 1999; Schuldberg, 2001). Individuals who are part of a university arts program can be thought of as creative because of their association with the program, their ability to meet admissions requirements, and general commitment to the arts (Frantom \& Sherman, 1999). In this study, creativity was also assessed by the Barron-Welsh Art Scale (BWAS). In a meeting where the ideas contained within this document were shared, the Associate Dean of the WVU CAC (Dr. William Winsor) agreed to assist in the recruitment of both students and faculty from that college to participate in the proposed study.

The term "artist" was defined in this dissertation as Feist (1999) operationalized it in his literature review. Specifically, it included visual artists (i.e., painters, sculptors, cinematographers, photographers, architects), literary artists (i.e., writers, poets), and performing artists (i.e., musicians, singers, dancers, actors). Samples comprised of artists who work in different mediums have been used by other researchers (Andreasen, 2008; Nowakowska et al., 2005; Santosa et al., 2007; Srivastava et al., 2010; Strong et al., 2007).

Chavez-Eakle et al. (2006) utilized a medium effect size in conducting a power analysis for their research. Therefore, a medium effect size was employed in determining a sample size for the current study. It was determined that a sample size of 76 participants was required given an alpha level (i.e., the probability of detecting an effect when one is not present or Type I error) 
of .05 and beta level (i.e., the probability of not finding a genuine effect that is present or Type II error) of .20 (Cohen, 1992; Field, 2005).

During the period between March, 2011 and September, 2011, 180 packets were administered to potential participants and 70 were returned to Dr. Windsor's office. After review of these packets, I determined that 60 of the 70 could be utilized in the data analysis. The returned packets that were excluded had one or more of the following problems; not all figures of the BWAS were rated by the participant, all of the figures on the BWAS were marked in one direction (i.e., the participant indicated he/she liked/disliked all 86 figures), items on the TCI-R were not rated, and demographic information was not provided (e.g., the participant did not provide his/her age).

In September, 2011 I consulted with my dissertation chair and committee members about utilizing a sample size that was smaller (i.e., 60 participants) than what I had proposed (i.e., 76 participants). Consensus was reached that I could commence with data analyses.

\section{Measures}

The Barron-Welsh Art Scale (BWAS). The BWAS has been empirically derived from the Welsh Figure Preference Test, which was an inventory of 400 black and white designs in a three by five inch format. These figures range from simplistic geometric forms to complex patterns. In addition, the figures vary in terms of symmetry, quality, and preparation (i.e., freehand vs. ruled preparation). In a pooled sample of 2,208 men and 1,005 women, 86 of these designs (which constitute the BWAS) were shown to consistently distinguish those with artistic potential and interests (e.g., art students, architects, playwrights, creative writers) from other individuals (e.g., business executives, military officers, college students majoring in engineering). People with artistic potential and interests have been found to typically perform at 
or above the mean score on the BWAS (i.e., known-groups validation or criterion-related validity). Odd-even coefficients (i.e., Split-Half Reliability) of the BWAS for multiple samples have ranged from .95 to .97 (Gough, Hall, \& Bradley, 1996). In addition, Gough, Hall, and Bradley (1996) reported that the Test-retest reliability coefficients (i.e., temporal stability) of the BWAS have been found to be at or above .90 . However, these authors were summarizing 40 years of research and did not provide the specific time intervals for specific Test-retest reliability coefficients.

Each item of the BWAS can be ranked as "like” or "dislike” by the respondent. This allows for scores on two subscales as well as a total score, which include BWAS-Like ("like” subscale total), BWAS-Dislike (“dislike” subscale total), and a BWAS-Total score. People with artistic potential and interests have generally been found to be high scorers on the BWAS-Total and typically have preferred complex (vs. simple), asymmetrical (vs. symmetrical), and freehand (vs. ruled) designs. The BWAS requires a combination of participant visual (observing the figures) and affective (determining whether each figure is liked/disliked) assessment of the designs. Thus, it has been purported to be a gauge of variability as well as flexibility in affect and cognition (Gough et al., 1996; Santosa et al., 2007; Strong et al., 2007). The aforementioned types of reliability and validity associated with the BWAS have been listed in Appendix B. Although the BWAS is copyrighted, I was given permission to include a sample page of this measure and it can be found in Appendix C.

The Temperament and Character Inventory - Revised (TCI-R). The TCI-R is comprised of four temperamental trait dimensions (i.e., Harm Avoidance, Novelty Seeking, Reward Dependence, and Persistence) and three character trait dimensions (i.e., SelfDirectedness, Cooperativeness, and Self-Transcendence). Each of the four temperament 
dimensions is comprised of four subscales while the three character dimensions are composed of five subscales (Cloninger, 2004; Cloninger, 2006; Cloninger et al., 1993; Cloninger et al., 1994; Gonclaves \& Cloninger, 2010). Descriptors of high and low scorers for each dimensional subscale are provided in Appendix A.

The TCI was originally validated in general community samples in 1993. Since that time, translations of the measure have been validated in other countries such as Japan, Germany, China, France, and Sweden. Convergent and concurrent validities of the assessment have also been evaluated by comparing it to the personality models of Millon, Coolidge, and various forms of five-factor models (Cloninger, 2008; Gonclaves \& Cloninger, 2010). Gonclaves and Cloninger (2010) summarized how extensively the TCI has been employed by stating that "the TCI and its translations have been used in thousands of peer-reviewed publications with many reproducible findings regarding genetics, neurobiology, brain imaging, learning, psychopharmacology, and clinical psychopathology” (p. 127).

The majority of research involving the TCI has involved the seven dimensions rather than the subscales of each dimension because these facets have demonstrated moderate internal consistency. However, accumulating clinical experience had revealed the potential utility of these subscales so the TCI-R was developed. The TCI-R represents three major modifications of the TCI. First, the number of subscales that assessed Reward Dependence and Persistence were increased without changing the conceptual construct of any of the seven major dimensions. Second, although the total number of items between the TCI and TCI-R are the same (i.e., 240 items), only 199 remain unchanged. Specifically, 37 items from the TCI were eliminated and 41 new items were added to the TCI-R (including 5 validity items). Third, the response format was changed from a true/false dichotomy such that the TCI-R has a 5 point Likert scale (i.e., 1- 
definitely false, 2-mostly false, 3-not true, 4-mostly true, 5-definitely true) (Gonclaves \& Cloninger, 2010).

The psychometric properties of the TCI-R have been evaluated in one American sample as well as seven samples from other countries. The Cronbach alpha coefficients (i.e., internal consistency reliability) of the seven dimensions were found to range from .71 to .92 in these studies. In addition, the range of Cronbach alpha coefficients for the subscales was .42 to .90 . The Cronbach alpha coefficients for the dimensions and subscales that were provided by the Gonclaves and Cloninger (2010) study are listed in Appendix A. The TCI-R subscales were not utilized in the current study due to the wide range of internal consistency. Due to the nonlinear relationship between the temperament and character dimensions, factor analysis of these two aspects of personality was performed by separate principal component analyses. All facets of the dimensions have generally loaded onto factors as expected with two exceptions (i.e., exploratory excitability, first facet of Novelty seeking; self-acceptance, fourth facet of Self-Directedness). However, these two subscales have loaded on factors other than their rationally defined dimensions since the psychometric properties of TCI have been investigated (Goncalves \& Cloninger, 2010). The aforementioned types of reliability and validity associated with the TCI-R are listed in Appendix B. Although the TCI-R is copyrighted, I was given permission to include a sample page of this measure and it can be found in Appendix D.

Demographic Questionnaire. I developed a demographic questionnaire (Appendix E) to gather information on variables that could represent covariates in the research design discussed below. These variables included gender, age, race, highest level of education, and major. No identifying information was collected. 


\section{Procedure}

This study was proposed and approved by the doctoral committee on December 6, 2010. I completed the WVU Institutional Review Board (IRB) mandated Collaborative Institutional Training Initiative on December 11, 2010. The WVU IRB research protocol was submitted and approved with exempt status on March 2, 2011. A cover letter to potential participants was generated as part of this process and can be found in Appendix F.

I met with Dr. Windsor a second time subsequent to being granted approval by the WVU IRB. The purpose of this meeting was to discuss the logistics of identifying participants for this study. It was decided that Dr. Windsor and the department heads of each of the three divisions of the WVU CAC (i.e., Art and Design, Music, Theatre and Dance) would identify classes of potential participants to distribute the packets of the three measures utilized in the study (discussed above). After the packets of measures were provided to willing participants in these identified classes, the professors of these classes returned the completed packets to Dr. Windsor's office where I later collected them.

\section{Research Design}

This study employed a between-subjects design. Participants were administered the demographic data questionnaire, the TCI-R, and the BWAS. The order of administration remained consistent. The sample was divided into three creative groups based on BWAS-Total score (i.e., low/medium/high scorers) by the following procedure. First, the mean (i.e., 26.50) and standard deviation (11.79) of the entire sample was identified. Second, half of the standard deviation (5.9) was added and subtracted from the mean to define the range of the medium 
creativity group (i.e., 21-32). The range of medium creativity group also served to define the upper limit of the low creativity group (i.e., 20) and the lower limit of the high creativity group (i.e., 33). Thus, the low creativity group had 19 participants and constituted a BWAS-Total score range of 5-20, the medium creativity group had 17 participants and constituted a BWAS-Total score range of 21-32, and the high creativity group had 24 participants and constituted a BWASTotal score range of 33-46.

In summary, scores on the BWAS-Total represented the independent/grouping variable of this study and the three groups constituted three levels of this variable. The three temperament and three character dimensions that contribute to temperament/character configurations represented the six dependent/outcome variables of the study. Configurations were based on where a participant scored in one of three percentile ranges (i.e., lowest one third, $0-33 \%$, middle one third, 34-66\%, and highest one third, 67-100\%).

Hypotheses and Analyses. Five preliminary multivariate analysis of variance (MANOVA) statistics were conducted to assess whether demographic variables (gender, age, race, education, and major) constituted covariates for the main analysis. In each of these analyses, one of the demographic variables represented the independent variable whereas the six TCI-R temperament and character trait scores were the dependent variables. In the main analyses, the different BWAS (i.e., creativity) groups were compared in terms of TCI-R temperament and character dimensions by use of a multivariate analysis of covariance (MANCOVA).

Hypothesis I: High scorers on the BWAS-Total will demonstrate significantly higher TCI-R scores than either of the other groups (i.e., Low or Medium scores on the BWAS-Total) 
on the following dimensions: Self-Directedness, Cooperativeness, Self-Transcendence, Reward Dependence, and Novelty Seeking.

Hypothesis II: High scorers on the BWAS-Total will evidence significantly lower TCI-R scores than the other two groups (i.e., Low or Medium scorers on the BWAS-Total) on the Harm Avoidance temperament dimension.

Thus, it was hypothesized that high scorers would exhibit the hypothesized "Creative" character configuration. In addition, it was purported they would endorse the "Passionate" (i.e., hyperthymic) temperament configuration rather than the "Reliable” (i.e., cyclothymic) temperament configuration (which only differ in terms of Novelty Seeking). This prediction was based on two previous findings. First, an association was found between affect and creativity as assessed by the Sensation Seeking Scale (SSS) in a sample of visual artists (Frantom \& Sherman, 1999). Second, the SSS has been found to correlate with low Harm Avoidance and high Novelty Seeking on the TCI-R (Cloninger et al., 1994). In sum, it was hypothesized that high scorers on the BWAS would be more likely to demonstrate a "Creative/Passionate" temperament/character configuration on the TCI-R than medium or low scorers on the BWAS. 


\section{Chapter 4: Results}

\section{Descriptive Statistics}

There were 60 participants who took part in the study. The majority of these individuals were white $(n=46,76.7 \%)$ and female $(n=32,53.3 \%)$. Many of the participants declared music as their major ( $\mathrm{n}=23,38.3 \%)$ and had a high school education but no higher degree $(\mathrm{n}=$ 25, 41.7\%). Frequencies and percentages for participant demographics are presented in Table 1.

Table 1

Frequencies and Percentages for Participant Demographics

Demographic $\quad n \quad \%$

Gender

Male

28

46.7

Female

32

53.3

Race

American Indian/Alaskan native

1

1.7

Asian

6

10.0

Black/African American

1

1.7

Native Hawaiian/Other Pacific Islander

1

1.7

White

46

76.7

Multiracial

5

8.3

Education 


\begin{tabular}{lcc}
\hline High School & 25 & 41.7 \\
BA/BS/BFA & 19 & 31.7 \\
MA/MS/MSW/MFA & 13 & 21.7 \\
PhD/PsyD/EdD/JD/MD & 3 & 5.0 \\
Major Groups & 18 & \\
Art \& Design & 23 & 30.0 \\
Music & 15 & 38.3 \\
Theatre \& Dance & 4 & 25.0 \\
Other & & 6.7 \\
Creativity Group & 19 & 31.7 \\
Low (5-20) & 17 & 28.3 \\
Medium (21-32) & 24 & 40.0 \\
High (33-46) & & \\
\hline
\end{tabular}

The youngest participant was 18 years old, and the oldest participant was 65 years old. On average participants were 32.48 years old $(S D=14.19)$. Means and standard deviations for Novelty Seeking, Harm Avoidance, Reward Dependence, Self-Directedness, Cooperativeness, and Self-Transcendence scores on the TCI-R are presented in Table 2. The reader will recall that scores for each dimension are percentile scores, and the temperament/character configurations are categorized based on where participants scored in one of three percentile ranges (i.e., lowest one third, 0-33\%, middle one third, 34-66\%, and highest one third, 67-100\%). 
Table 2

Means and Standard Deviations for Temperament and Character Percentile Scores

\begin{tabular}{lcc}
\hline Variable & $M$ & $S D$ \\
\hline & & \\
Novelty Seeking & 55.53 & 28.0 \\
Harm Avoidance & 61.58 & 28.8 \\
Reward Dependence & 45.53 & 30.2 \\
Self-Directedness & 40.25 & 29.5 \\
Cooperativeness & 52.75 & 31.6 \\
Self-Transcendence & 53.80 & 32.4 \\
\hline
\end{tabular}

\section{Hypothesis 1}

High scorers on the BWAS-Total will demonstrate significantly higher TCI-R scores than either of the other groups (i.e., Low or Medium scorers on the BWAS-Total) on the following dimensions: Self-Directedness, Cooperativeness, Self-Transcendence, Reward Dependence, and Novelty Seeking.

To examine Hypothesis 1, a multivariate analysis of covariance (MANCOVA) was conducted to assess if there were differences in Self-Directedness, Harm Avoidance, Cooperativeness, Self-Transcendence, Reward Dependence, and Novelty Seeking by the creativity group to which they were classified after controlling for certain demographic variables. The assumption for normality was assessed with six Kolmogorov Smirnov tests. The results of these tests were significant for only Harm Avoidance, which constituted a violation of 
the assumption of normality. However, non-normality has only a slight affect on Type I error (Stevens, 2009). The assumption of absence of multicollinearity was assessed by examining a Pearson correlation matrix between the dependent variables. No correlations between variables were above \pm .80 so multicollinearity was considered absent.

The demographics that were assessed to be covariates were gender, age, race, education, and academic major groups. Age was assessed and used as a continuous variable when determining whether it would be a covariate such that no age groups were created. Race was dichotomized into white and not white categories due to the lack of diversity within the sample. Table 1 contains the educational categories that were assessed. Due the variety of majors in the sample, four major categories (also listed in Table 1) were created so that this potential covariate could be evaluated.

Results of testing for covariates by preliminary MANOVAs showed that race and education were significantly related to Self-Directedness, Cooperativeness, Self-Transcendence, Reward Dependence, Harm Avoidance, and Novelty Seeking and should be used as covariates in the MANCOVA. Two statistically significant relationships were identified. First, white individuals $(M=60.26, S D=28.27)$ scored statistically higher than the minority group $(M=$ 28.07, $S D=30.20)$ on Cooperativeness. Second, the minority group $(M=66.50, S D=26.70)$ scored statistically higher than white participants $(M=49.93, S D=33.29)$ on SelfTranscendence.

The assumption of equality of covariance could not be assessed by the Box’s Test of Equality of Covariance Matrices due to the unequal number of participants in the three creativity groups. However, Levene's Test of Equality of Error Variances was conducted for each of the dependent variables. All tests were non-significant indicating that the assumption of equality of 
variances across the TCI-R dimensions. The Pillai's Trace statistic was used to assess significance of the MANCOVA since this statistic is most robust to violation of assumptions when group sizes are unequal (Field, 2005). Results of the MANCOVA were not significant for creativity group, $F(14,96)=1.53, p=.114$. This suggests that Self-Directedness, Cooperativeness, Self-Transcendence, Reward Dependence, and Novelty Seeking were not significantly different by creativity group, after controlling for education and race. Thus, the null hypothesis cannot be rejected in favor of the alternative hypothesis. Results for the MANCOVA are presented in Table 3. Means and standard deviations for Self-Directedness, Cooperativeness, Self-Transcendence, Reward Dependence, Harm Avoidance, and Novelty Seeking by creativity group are presented in Table 4.

\section{Table 3}

\section{Means and Standard Deviations for Temperament and Character Percentile Scores}

Results for the MANCOVA for Self-Directedness, Cooperativeness, Self-Transcendence, Reward Dependence, Harm Avoidance, and Novelty Seeking by Creativity Group Controlling for Age, Gender, and Race

\section{ANOVA $F$}

\begin{tabular}{lccccccc} 
Source & MANCOVA F & NS & HA & RD & SD & CO & ST \\
\hline Education & $1.86^{*}$ & 1.94 & 0.90 & 0.89 & 1.03 & 0.50 & 0.72 \\
Race & $3.84^{* *}$ & 2.62 & 1.44 & 3.18 & 3.52 & $10.14^{* *}$ & $4.73^{*}$ \\
Creativity Group & 1.53 & 0.41 & 0.26 & 0.28 & 0.87 & 0.28 & 2.65 \\
\hline
\end{tabular}

Note. NS = Novelty Seeking. HA = Harm Avoidance. RD = Reward Dependence. SD = SelfDirectedness. $\mathrm{CO}=$ Cooperativeness. ST $=$ Self-Transcendence.

$* p<0.05$. ** $p<0.01$. 


\section{Table 4}

Means and Standard Deviations for Self-Directedness, Cooperativeness, Self-

Transcendence, Reward Dependence, Harm Avoidance, and Novelty Seeking by Creativity Group

\begin{tabular}{lcccccc}
\hline & \multicolumn{2}{c}{ Low (5-20) } & \multicolumn{2}{c}{ Medium (21-34) } & \multicolumn{2}{c}{ High (35-46) } \\
& $M$ & $S D$ & $M$ & $S D$ & $M$ & $S D$ \\
& & & & & & \\
& & & & & & \\
Novelty Seeking & 52.84 & 22.17 & 61.41 & 32.79 & 53.50 & 29.06 \\
Harm Avoidance & 59.21 & 28.40 & 62.18 & 29.81 & 63.04 & 29.44 \\
Reward Dependence & 43.89 & 31.13 & 44.47 & 31.13 & 47.58 & 30.04 \\
Self-Directedness & 39.58 & 29.98 & 44.76 & 28.00 & 37.58 & 31.10 \\
Cooperativeness & 57.32 & 30.43 & 54.59 & 31.97 & 47.83 & 32.89 \\
Self-Transcendence & 64.37 & 30.65 & 46.29 & 31.14 & 50.75 & 33.86 \\
\hline
\end{tabular}

\section{Hypothesis 2}

High scorers on the BWAS-Total will evidence significantly lower TCI-R scores than the other two groups (i.e., Low or Medium scorers on the BWAS-Total) on the Harm Avoidance temperament dimension.

Since the null hypothesis was accepted instead of Hypothesis 1, Hypothesis 2 was not examined. 


\section{Chapter 5: Discussion}

Rigorous empirical investigation may eventually establish a relationship between creativity and mental health rather than mental illness (Andreasen, 2008; Prentky, 2001; Richards, 2001; Rothenberg, 2001; Waddell, 1998). I believe a person’s creativity can be associated with positive aspects of his/her mental health regardless of whether he/she manifests symptoms of psychological pathology. This is consistent with one of the five unifying themes of counseling psychology (i.e., emphasis on a person's assets and strengths) as identified by Gelso and Fretz (2001). Therefore, I reviewed the empirical literature relating to the proposed relationship between creativity and the bipolar disorders from the perspective of a counseling psychology strength/asset focused perspective.

The putative association between bipolarity and creativity is flawed to the extent that it assesses some aspects of the bipolar spectrum of disorders (e.g., bipolar disorder I, bipolar disorder II) but excludes more subtle parts of the bipolarity continuum (e.g., sub-syndromal symptoms of hypomania, different types of temperament). There is need for research that identifies how different groups of temperamental traits are related to aspects of creativity (Chavez-Eakle et al., 2006; Mumford, 2003; Nowakoska et al., 2005). Thus, the research question of this dissertation: Are the creative advantages associated with the milder part of the bipolar continuum due to a specific type (or types) of temperament?

I chose the psychobiological model of temperament and character to provide a theoretical context for which to consider the possible relationship between traits and aspects of creativity. Two hypotheses were proposed to address the research question. The first hypothesis stated that high scorers on the Barron-Welsh Art Scale Total Score (BWAS-Total) would demonstrate 
significantly higher scores on the Temperament and Character Inventory - Revised (TCI-R) than either of the other groups (i.e., Low or Medium scorers on the BWAS-Total) on the following dimensions: Self-Directedness, Cooperativeness, Self-Transcendence, Reward Dependence, and Novelty Seeking. The second hypothesis proposed that high scorers on the BWAS-Total would evidence significantly lower TCI-R scores than the other two groups (i.e., Low or Medium scorers on the BWAS-Total) on the Harm Avoidance temperament dimension. Essentially, participant BWAS-Total scores were the independent/grouping variable whereby they were placed into one of three groups by adding/subtracting a half of a standard deviation to/from the sample mean in order to define the range of the medium group (as well as the parameters of the low and high groups). The six variables of temperament and character constituted outcome/dependent variables.

The multivariate analysis of covariance (MANCOVA) was non-significant such that the creativity groups could not be differentiated by any of the TCI-R dimensions. Before reviewing the possible influences of the non-significant finding, I will address the two significant relationships associated with the covariates of the MANCOVA.

Both significant findings relate to differences between white participants and participants of color. Neither of these relationships will be interpreted due to the fact that the sample of this study was predominantly white (i.e., 76.7\%) and that people of color were placed into one group in order to be able to render any type of statistical comparison on this demographic variable. As such, it is likely the myriad differences that exist between different non-white participants cannot be examined. Furthermore, it does not seem meaningful to attempt to interpret differences between two groups when one group (i.e., the minority group) represents 
a relatively small percentage of the overall sample and consists of participants who may differ from each other as much as they are different from the other group.

The rejection of the two hypothesis of this study will now be discussed. Sun, Pan, and Wang (2011) recommended that when significant findings are not obtained, a researcher should evaluate the potential influence of three possible contributors to the necessity of accepting the null hypothesis. They specified these points of consideration as the power of the study (including the proposed effect size), attributes/appropriateness of instruments employed, and the research design. First, limitations relating to the power of this study will be delineated. Second, the implications/limitations associated with the utilization of the BWAS as well as other aspects of the research design will be reviewed.

The low statistical power of this study increases the likelihood of rejecting the research hypotheses in favor of the null hypothesis and constitutes a potential Type II error. This is certainly due in part to not achieving the proposed sample size in the a priori power analysis. However, I contend that the effect size I employed in this research (i.e., medium effect size), which was based on the Chavez-Eakle et al. (2006) study, may have been larger than the actual effect size in the population sampled. This position derives from the literature review above and can inform future research. Therefore, I will first review the tenets of power analysis in order to provide a context in which to offer support for this argument.

Statistical power is a function of the particular statistical test employed, directionality of the statistical test, alpha level (i.e., likelihood of falsely rejecting the null hypothesis, or Type I error), beta level (i.e., likelihood of falsely rejecting the alternative hypothesis, or Type II error), effect size (i.e., the magnitude of the discrepancy between the null hypothesis and the alternative hypothesis that exists in a population of interest), and number of participants (Balkin \& Sheperis, 
2011; Cohen, 1992a; Cohen, 1992b; Jones \& Sommerlund, 2007; Heppner, Wampold, \& Kivlighan, 2008; Sun et al., 2011). In other words, "the statistical power of a significance test is the long-term probability, given the population effect size, alpha level, and sample of rejecting the null hypothesis” (Cohen, 1992a, p. 56).

While some of the aforementioned aspects of a power analysis are established, others are variable. The type and directionality of statistical test are dictated by the research design while the alpha and beta levels are generally set by convention (Balkin \& Sheperis, 2011; Cohen, 1992a; Cohen, 1992b; Jones \& Sommerlund, 2007; Heppner et al., 2008). However, effect size and sample size have a relationship such that, "neither power nor sample size can be determined in the absence of the investigator's readiness to consider just how wrong the null hypothesis is likely to be (i.e., the effect size)" (Cohen, 1992b, p. 99). Therefore, a researcher must accurately estimate the effect size of the population of interest prior to conducting a power analysis in determining an appropriate sample size. This is considered the most challenging aspect of performing a power analysis (Balkin \& Sheperis, 2011; Cohen, 1992a; Cohen, 1992b; Jones \& Sommerlund, 2007; Heppner et al., 2008; Sun et al., 2011).

Heppner et al. (2008) outlined two ways investigators generally identify an effect size for the population they are interested in sampling. First, effect sizes from prior studies can be utilized. However, the reliability of this approach can be challenged due to the fact that the majority of social science research has yet to incorporate power analyses (Balkin \& Sheperis, 2011; Sun et al., 2011). As a result, there is generally a modicum of knowledge regarding the magnitude of difference between null and alternative hypotheses in most populations of psychological research (Balkin \& Sheperis, 2011; Cohen, 1992a; Cohen, 1992b; Jones \& Sommerlund, 2007; Heppner et al., 2008; Sun et al., 2011). 
A second method entails purporting what effect size would be sufficiently large to have practical and/or clinical significance (Heppner et al., 2008). This is often accomplished via the categorical system of small, medium, and large effect sizes (Balkin \& Sheperis, 2011; Jones \& Sommerlund, 2007; Heppner et al., 2008; Sun et al., 2011). This system was created by Jacob Cohen and was designed such that small effect sizes were large enough to be relevant (Cohen, 1992b). Although these classifications are helpful, researchers still have to stipulate which of the categories a particular study will be able to detect (Heppner et al., 2008).

The only study of the literature reviewed that provided specific information on power analysis was the Chavez-Eakle et al. (2006) study. In addition, the series of articles from the research group at Stanford University (i.e., Nowakowska et al., 2005; Santosa et al., 2007; Srivastava et al., 2010; Strong et al., 2007) cited low power as a limitation of their studies. Thus, an important shortcoming of the research investigating the association between bipolarity/temperament and creativity is the dearth of knowledge pertaining to the effect size of this particular population. In conducting the power analysis for the current study, a medium effect size was chosen because that is what Chavez-Eakle et al. (2006) utilized in their work. In addition, it is common practice for researchers to identify a medium effect size in the absence of available information relating to their selected population (Heppner et al., 2008).

In retrospect, there are differences between the population Chavez-Eakle et al. (2006) sampled and the population sampled for the current study. The creativity group in Chavez-Eakle et al. (2006) consisted of 30 individuals who were members of the National System of Researchers or Creators in Mexico. The primary purpose of these organizations is described by the authors as providing support to the most prolific scientists and artists in Mexico. For example, members of these organizations are required to maintain continuous and successful 
achievement in their respective fields. In comparing this creativity group to the sample of the current study, the reader will recall the previous discussion about possible differences between research of eminent (i.e., big C) and everyday (i.e., little c) creativity. Specifically, there is dissention as to whether these two populations are qualitatively or quantitatively distinct (Baer \& Kaufman, 2006; Feldhusen \& Goh, 1995; Plucker et al., 2004; Richards et al., 1988; Richards \& Kinney, 1990; Santosa et al., 2007; Simonton, 2002; Smith, 2005). As such, it is possible the population Chavez-Eakle et al. (2006) studied has a medium effect size whereas the population of the current study has a small effect size.

Context-dependent mediation is a threat to external validity that further underscores the possible differences in population effect sizes. This term refers to the possibility that a mediating relationship may exist for one group of individuals (i.e., context) but not for another (Heppner et al., 2008). For example, the population of people who possess everyday creativity may be a more heterogeneous group than those who are considered eminently creative. Not only does this potentially greater variability in the everyday creativity population increase the likelihood of an unidentified mediating relationship(s), but it also introduces more variance in how they respond to assessments. In general, heterogeneity of participants increases error variance and contributes to less powerful statistical tests (Balkin \& Sheperis, 2011; Benham, 2005; Heppner et al., 2008).

The sample of this study was heterogeneous due to the fact that the participants were engaged in a variety of artistic disciplines. This could have contributed to error variance and constitutes a statistical conclusion validity threat. However, as noted in chapter three, the use of samples that are comprised of artists who work in different mediums is commonplace in the research that investigates the association between bipolarity/temperament and creativity (Andreasen, 2008; Chavez-Eakle et al., 2006; Nowakowska et al., 2005; Santosa et al., 2007; 
Srivastava et al., 2010; Strong et al., 2007). Perhaps this practice has contributed to a lack of findings even in studies that have revealed some significant relationships between bipolarity/temperament and creativity.

The decision to utilize the BWAS was a limitation of the study for several reasons that will now be discussed. The reader will recall I chose the BWAS as my measure of creativity for two reasons. In previous research, it demonstrated a relationship with traits of hypomania and had been found to assess facets of creativity that are distinct from other instruments (Nowakowska et al., 2005; Santosa et al., 2007; Schuldberg, 2001; Srivastava et al., 2010; Strong et al., 2007).

Santosa et al. (2007) found that their bipolar disorder (BP) and creative control (CC) groups could be differenced from their healthy control group via BWAS scores. The other creativity measures employed in this study were not able to make this distinction. However, in the authors' discussion of their study limitations, they acknowledged that "statistical power limitations could account for some of our negative findings, and particularly limit our ability to assess creativity in the subgroups of the CC and BP groups” (Santosa et al., 2007, p. 37). Thus, it is possible some of the other creativity measures utilized in their study had a significant relationship with the BP and/or CC groups that were unrecognized due to low power. In addition, the commonalities between the CC and BP groups (and thus how they were found to be distinguished by the BWAS) could be due to how these groups were undifferentiated in terms of possible trait symptoms (i.e., many of the participants in the CC group met criteria for a past psychiatric disorder).

The potential for other measures of creativity to form groups that could reveal the hypothesized TCI-R temperament/character configurations is further suggested by the 
Schuldberg (2001) finding that the majority of the variance of several creativity instrument scores was accounted for by hypomanic traits. However, this research may have been overpowered since the author pooled data from several of his previous studies to create a sample of 1,108 participants. In regard to the use of large samples, Jones and Sommerland (2007) stated, "in a study with a large sample size, group differences which are found to be statistically significant could in terms of magnitude (effect size) be meaningless” (p. 229).

The use of only one measure of creativity is a limitation of this study. Despite the identified power concerns of the aforementioned studies, each employed multiple instruments of creativity en route to discovering a significant finding. Mono-operation (i.e., use of a single creativity instrument) introduces bias when measuring multifaceted constructs by under representing the phenomena of interest (Heppner et al., 2008). This is certainly relevant for creativity research as authors generally agree the construct incorporates many possible elements, which renders it difficult to identify and define (Baer \& Feldhusen \& Goh, 1995; Kaufman, 2006; Sternberg, 2006).

A similar threat to construct validity that is relevant to this study is mono-method bias (Heppner et al., 2008). The BWAS is a self-report assessment and the power of this research could have been enhanced by introducing a different type of creativity measure. This is a limitation of the extant literature in general as all the studies reviewed incorporated self-report instruments. This is why many authors have attempted to develop various systems for creative product assessments but none have been able to establish sufficient criterion validity or operationalize how such products would be judged (Makel \& Plucker, 2008).

Self-report measures are often associated with the additional construct validity threats of participant hypothesis guessing and evaluation apprehension (Heppner et al., 2008). As such, 
additional limitations of this study relating to the use of the BWAS include the possibility that some participants based their answers on what they believed the current study was attempting to find or what they considered to be socially desirable answers.

These concerns about employing the BWAS as a gauge of some facets of creativity may also detract from the validity of the groups that were established and compared in this study. In other words, it may have been preferable to compare a creative group to a control group rather than using a single measure to distinguish levels of creativity.

Various types of selection bias represent a final limitation of this study. The reader will recall that 70 of 180 (38.9\%) packets were returned and only 60 (33.3\%) of these completed packets could be used in the analyses. It could be that potential participants who did embody the purported temperament and character configuration chose not to participate in the study due to a shared attribute. In addition, previous research has found that volunteer participants differ from those who do not volunteer. Characteristics that have distinguished these two groups suggest that volunteers are better educated and have a higher need for social approval (Heppner et al., 2008). Another possibility is that the subgroup of packets that were returned but not included due to various deficiencies represented a group of individuals that systematically differed from those who were incorporated in the sample.

\section{Future Research}

Many of the limitations of the present study that were discussed above could be utilized to inform future research on the purported relationship between bipolarity/temperament and facets of creativity. For example, future studies should focus on one type of artist and employ multiple measures of creativity. The only study reviewed that contains both of these elements is the research of Frantom and Sherman (1999). The reader will recall these researchers sampled 
visual artists and employed two creativity instruments. Interestingly, they found a significant relationship between creativity (as assessed by their two measures) and characteristics that are consistent with a hyperthymic temperament (i.e., strong need for stimulation, excitement, complex sensations and experiences, and maintenance of an optimum level of arousal).

Researchers may be more likely to identify differences in temperament if they compare participants who identify as creative to participants who do not rather than utilizing one or more creativity measures to divide their sample into levels of creative groups. The employment of a social desirability measure may reduce the likelihood of this construct validity threat impacting findings. In a similar vein, refining and using some type of product assessment could enable a more comprehensive assessment of facets of creativity that potentially have a relationship with temperament.

Investigators should identify whether they intend to sample a population that demonstrates everyday creativity or can be considered eminently creative. This is important given my contention that the former population may have a small (but not trivial) effect size while the latter population likely has a medium effect size. Furthermore, since an anticipated small effect size requires a larger sample than an anticipated medium effect size (Cohen, 1992a; Cohen 1992b; Jones \& Sommerlund, 2007), researchers should consider how they will be able to recruit a sufficient number of participants. Perhaps providing incentives to participate could help reduce various forms of selection bias.

Sun et al. (2011) argued that the importance of replication has not been emphasized enough in social science research. They attribute this deficiency to both editorial preference for originality and the difficulty associated with exact replication. However, studies that utilize the same sampling method and research design are possible (Sun et al., 2011). This is important 
because the concept of replication is critical to the methodology of sampling distributions, Type I/II errors, and null hypothesis testing. In other words, the results of a single study provide limited information whereas the essence of a topic of investigation emerges over repeated samples. Furthermore, insufficient knowledge of effect size in relation to level of significance cultivates a potential for serious errors in statistical inference and theory development (Balkin \& Sheperis, 2011; Heppner et al., 2008; Jones \& Sommerlund, 2007; Sun et al., 2011). Cognizance of these methodological principles can likely help resolve inconsistent and conflicting findings within the literature pertaining to the purported relationship between bipolarity/temperament and creativity.

Cohen (1992b) identified meta-analysis as a second form of power analysis in that it can assist in identification of a hypothesized effect size for a specified sample and alpha level. Sun et al. (2011) indicated that meta-analytic technique is not overly influenced by statistical significance of individual studies but instead it "emphasizes the need to explicitly design and place the studies in the context of the effects of prior research and compare the new results to the previous findings” (p. 86). Moreover, meta-analysis studies can be used by researchers as a guide for their research design (Cohen, 1992b; Sun et al., 2011). It is for these reasons that the body of research relating to bipolarity/temperament and creativity could benefit from a meta-analysis.

\section{Summary and Conclusions}

Many individuals throughout history have speculated about a possible relationship between creativity and mental illness (Becker, 2001; Prentky, 2001; Simonton, 1999; Waddell, 1998). The purported relationship between mental illness and creativity has been most heavily researched in individuals diagnosed with affective disorders (particularly the bipolar disorders) (Akiskal \& Akiskal, 1988; Akiskal, Savino, \& Akiskal, 2005; Andreasen \& Glick, 1988; 
Ghardirian, Gregoire, \& Kosmidis, 2000; Santosa et al., 2007; Schuldberg, 2001; Srivastava et al., 2010; Waddell, 1998). Disagreement persists about whether conclusions about this potential link can be offered (Andreasen, 2008; Benham, 2005; Chavez-Eakle et al., 2006; Frantom \& Sherman, 1999; Ghadirian et al., 2000; Kinney \& Richards, 2007; Makel \& Plucker, 2008; Rothenberg, 2001; Waddell, 1998).

Some authors have argued that the majority of studies that investigate the relationship between creativity and individuals diagnosed with bipolar disorder are plagued by problems in sampling, methodology, presentation of results, and/or conclusions (Andreasen \& Glick, 1988; Andreasen, 2008; Prentky, 2001; Richards \& Kinney, 1990; Rothenberg, 2001; Santosa et al., 2007; Strong et al., 2005; Waddell, 1998). For example, there is minimal knowledge regarding the effect size of the creative population. Furthermore, it is likely that there are differing effect sizes for individuals who engage in everyday creativity as compared to those who are eminently creative.

Despite the aforementioned limitations of the literature, there is accumulating empirical support that individuals who experience milder forms of the bipolar spectrum are more likely to be more creative than those who suffer from the more severe variants of the spectrum (Frantom \& Sherman, 1999; Ghardirian et al., 2000; Kinney \& Richards, 2007; Richards, 2001; Simonton, 1999; Waddell, 1998). Specifically, traits consistent with hypomania, rather than a full hypomanic episode, have been linked to aspects of creativity. In addition, some authors have hypothesized that individuals diagnosed with cyclothymic disorder may possess a creative advantage because their moods are moderately intense relative to those who experience hypomania or mania (Akiskal \& Akiskal, 1988; Akiskal, Savino, et al., 2005; Andreasen, 2008; 
Richards, 2001; Simonton, 2002). It is also possible that a non-pathological aspect of the bipolar spectrum (i.e., hyperthymic temperament) could contribute to enhanced creativity.

The current study did not find a significant relationship between creativity (as assessed by the BWAS) and a hyperthymic (i.e., TCI-R "passionate" configuration) or cyclothymic (i.e., TCI-R "reliable" configuration) temperament. However, the limitations outlined above may have detracted from overall statistical power of my research. Thus, perhaps the greatest value of this study for future investigations is the suggestions for future research that derive from my review of the literature and the acknowledged limitations of my research design. Ultimately, statistical hypothesis testing and power analyses are based on replication and there has not been nearly enough research to render definitive conclusions about the relationship between bipolarity/temperament and creativity.

It is also important to remain cognizant of the high likelihood that creativity emerges from several interactive facets (e.g., motivation, perception, action, temperament, social interaction, environment, etc.). Moreover, the process of how these components interact is probably best characterized as a nonlinear dynamic system with a variety of manifestations (Caselli, 2009; Plucker et al., 2004; Schuldberg, 2001). This is likely why the creativity construct has been associated with a multitude of conflicting traits (Galang, 2010; Qian et al., 2010). Therefore, temperament is just one aspect of the creativity matrix. It may be shortsighted for researchers to investigate one aspect of the multifaceted (and interdependent) construct while disregarding other interrelated facets of the phenomenon. In other words, specific temperaments are likely not causal agents per se but instead serve to lower behavioral thresholds, which increase the likelihood of an individual engaging in a creative act (Feist \& Barron, 2003). 
A comprehensive theory of the multifaceted construct of creativity may be necessary to guide how the phenomenon is studied. This is true for the creativity literature in general and is particularly relevant to the research that investigates the association between bipolarity/temperament and creativity. It may be that the psychobiological model of temperament and character can contribute to the understanding of the temperament aspect of creativity if it is examined in conjunction with other elements of the construct. Furthermore, there are specific components of creativity that need to be better understood before a comprehensive theory can be offered. For example, the reader will recall that the impact of environmental influences on what is considered creative is becoming increasingly recognized as another aspect of the construct (Baer \& Kaufman, 2006; Makel \& Plucker, 2008 Mumford, 2003). However, a psychometrically acceptable measure of environmental influence on creativity has yet to be established. Moreover, specific environmental variables that facilitate/inhibit creativity need to be determined (Makel \& Plucker, 2008). Therefore, as Sternberg (2006) suggested, the construct of creativity must be subject to ongoing scientific inquiry. 


\section{References}

Akiskal, H.S. (2004). The bipolar spectrum in psychiatric and general medical practice. Primary Psychiatry, 11(9), 30-35.

Akiskal, H.S. \& Akiskal, K.K. (1988). Reassessing the prevalence of bipolar disorders: Clinical significance and artistic creativity. Psychiatrie \& Psychobiologie, 3, 29-36.

Akiskal, H.S., Akiskal, K.K., Radwan, F., Haykal, J., Manning, S., \& Connor, P.D. (2005).

Temps-a: Progress towards validation of a self-rated clinical version of the temperament evaluation of the Memphis, pisa, paris, and san diego autoquestionnaire. Journal of Affective Disorders, 85, 3-16.

Akiskal, H.S., Mendlowicz, M.V., Girardin, J.L., Rapaport, M.H., Kelsoe, J.R., Gillin, J.C., \& Smith, T.L. (2005). Temps-a: Validation of a short version of a self-rated instrument designed to measure variations in temperament. Journal of Affective Disorders, 85, 4552.

Akiskal, K.K., Savino, M., Akiskal, H.S. (2005). Temperament profiles in physicians, lawyers, managers, industrialists, architects, journalists, and artists: A study in psychiatric outpatients. Journal of Affective Disorders, 85, 201-205,

Allen, R. \& Smith, D.J. (2008). Screening for bipolar disorder: Strengths and limitations of currently available instruments. Primary Care and Community Psychiatry, 13(2), 47-51.

American Psychiatric Association (2000). Diagnostic and statistical manual of mental disorders ( $4^{\text {th }}$ ed. text revision). Washington, DC: Author.

Andreasen, N.C. (2008). The relationship between creativity and mood disorders. Dialogues in Clinical Neuroscience, 10(2), 251-255. 
Andreasen, N.C., Endicott, J., Spitzer, R.L., \& Winokur, G. (1977). The family history method using diagnostic criteria. Reliability and validity. Archives of General Psychiatry, 34, 1229-1235.

Andreasen, N., \& Glick, I. (1988). Bipolar affective disorder and creativity: implications and clinical management. Comprehensive Psychiatry, 29(3), 207-217.

Averill, J.R. (2002). Emotional creativity. In C.R. Snyder \& S.J. Lopez (Eds.), Handbook of positive psychology (pp. 172-185). New York: Oxford University Press.

Bader, C.D. \& Dunner, D.L. (2007). Bipolar disorder not otherwise specified in relation to the bipolar spectrum. Bipolar Disorders, 9, 860-867.

Baer, J. \& Kaufman, J.C. (2006). Creativity research in english-speaking countries. In J.C. Kaufman \& R.J. Sternberg (Eds.), The International Handbook of Creativity (pp. 11-37). New York: Cambridge University Press.

Balkin, R.S. \& Sheperis, C.J. (2011). Evaluating and reporting statistical power in counseling research. Journal of Counseling and Development, 89, 268-272.

Barron, F.X. \& Harrington, D.M. (1981). Creativity, intelligence, and personality. Annual Review of Psychology, 32, 439-476.

Becker, G. (2001). The association of creativity and psychopathology: Its cultural-historical origins. Creativity Research Journal, 13(1), 45-53.

Benham, C. (2005). The relationship between creativity and psychiatric vulnerability: A metaanalysis of empirical studies. Dissertation Abstracts International, 65, Retrieved from PsychINFO database. 
Bolling, M. (2004). Subtle psychological side effects: Documentation, description, and treatment implications of subjective experiences of selective serotonin reuptake inhibitors taken for depression. Dissertation Abstracts International, 64, Retrieved from PsycINFO database.

Brewer, M.B. \& Chen, Y. (2007). Where (who) are collectives in collectivism? Toward conceptual clarification of individualism and collectivism. Psychological Review, 114(1), $133-151$.

Burgess, S., Geddes, J., Hawton, K. Townsend, E., Jamison, K., \& Goodwin, G. (2001). Lithium for maintenance treatment of mood disorders. Cochrane Database of Systematic Reviews, $3,1-17$.

Caselli, R. (2009). Creativity: an organizational schema. Cognitive and Behavioral Neurology: Official Journal Of The Society For Behavioral And Cognitive Neurology, 22(3), 143154.

Chavez-Eakle, R.A., del Carmen Lara, M., \& Cruz-Fuentes, C. (2006). Personality: A possible bridge between creativity and psychopathology? Creativity Research Journal, 18(1), 2738.

Cloninger, C.R. (1987). A systematic method for clinical description and classification of personality variants. Archives of General Psychiatry, 44, 573-588.

Cloninger, C.R. (2004). Feeling good: The science of well-being. New York: Oxford University Press.

Cloninger, C.R. (2006). Differentiating personality deviance, normality, and well-being by the seven-factor psychobiological model. In. S. Strack (Ed.), Differentiating normal and abnormal personality ( $2^{\text {nd }}$ ed.) (pp. 65-81). New York, NY: Springer Publishing Co. 
Cloninger, C.R. (2008). The psychobiological theory of temperament and character: Comment on Farmer and Goldberg (2008). Psychological Assessment, 20(3), 292-299.

Cloninger, C.R., Przybeck, T.R., Svrakic, D.M., \& Wetzel, R.D. (1994). The temperament and character inventory: A guide to its development and use. St. Louis, MO: Washington University Center for Psychobiology of Personality.

Cloninger, C.R., Svrakic, D.M., \& Przybeck, T.R. (1993). A psychobiological model of temperament and character. Archives of General Psychiatry, 50(9), 975-990.

Cloninger, C.R., Svrakic, N.M., \& Svrakic, D.M. (1997). Role of personality self-organization in development of mental order and disorder. Development and Psychopathology, 9, 881906.

Cohen, J. (1992a). A power primer. Psychological Bulletin, 112, 155-159.

Cohen, J. (1992b). Statistical power analysis. Current Directions in Psychological Science, 1(3), 98-101.

Eysenck, H.J. (1995). Creativity as a product of intelligence and personality. In D.H. Saklofske \& M. Zeidner (Eds.), International Handbook of Personality and Intelligence (pp. 231245), New York: Plenum Press.

Faber, D. (2003). The criterion and construct validity of the performance-based personality assessment of C.R. Cloninger's temperament and character inventory. Dissertation Abstracts International, 63, Retrieved from PsychINFO database.

Feist, G.J. (1999). The influence of personality on artistic and scientific creativity. In R.J. Sternberg (Ed.), Handbook of Creativity (pp. 273-296), New York, NY: Cambridge University Press. 
Feist, G.J. \& Barron, F.X. (2003). Predicting creativity from early to late adulthood: Intellect, potential, and personality. Journal of Research in Personality, 37, 62-88.

Feldhusen, J.F. \& Goh, B.E. (1995). Assessing and accessing creativity: An integrative review of theory, research, and development. Creativity Research Journal, 8(3), 231-247.

Field, A. (2005). Discovering statistics using spss. Thousand Oaks, CA: Sage Publications.

Frantom, C., \& Sherman, M. (1999). At what price art? Affective instability within a visual art population. Creativity Research Journal, 12(1), 15-23.

Galang, A.J. (2010). The prosocial psychopath: Explaining the paradoxes of the creative personality. Neuroscience and Biobehavioral Reviews, 34, 1241-1248.

Gelso, C. \& Fretz, B. (2001). Counseling psychology ( $2^{\text {nd }}$ Ed). Belmont, CA: Thompson/Wadsworth.

Ghadirian, A., Gregoire, P., \& Kosmidis, H. (2000). Creativity and the evolution of psychopathologies. Creativity Research Journal, 13(2), 145-148.

Goncalves, D.M. \& Cloninger, C.R. (2010). Validation and normative studies of the brazilian Portuguese and American versions of the temperament and character inventory - revised (TCI-R). Journal of Affective Disorders, 124, 126-133.

Goodwin, F.K. \& Jamison, K.R. (1990). Manic-depressive illness. New York: Oxford University Press.

Gough, H.G., Hall, W.B., \& Bradley, P. (1996). Forty years of experience with the barron-welsh art scale. In A. Montuori (Ed), Unusual Associates: A Festschrift for Frank Barron (p. 253-285). Cresskill, NJ: Hampton Press.

Heppner, P.P., Wampold, B.E., \& Kivlighan, D.M. (2008). Research design in counseling. New York: Thompson Brooks Cole Press. 
Horne, R. (1998). Adherence to medication: A review of existing research. In L.B. Myers \& K. Midence (Eds.), Adherence to treatment in medical conditions (pp. 285-310). Amsterdam Netherlands: Harwood Academic Publishers.

Horne, R., \& Weinman, J. (1998). Predicting treatment adherence: An overview of theoretical models. In L.B. Myers \& K. Midence (Eds.), Adherence to treatment in medical conditions (pp. 25-50). Amsterdam Netherlands: Harwood Academic Publishers.

Jamison, K. (1990). Medication compliance. In F. Goodwin \& K. Jamison (Eds.), Manic depressive illness (pp. 746-762). Oxford: Oxford University Press.

Jamison, K. (1993). Touched with fire: Manic-depressive illness and the artistic temperament. New York, NY: Free Press.

Jamison, K., Gerner, R., \& Goodwin, F. (1979). Patient and physician attitudes toward lithium: Relationship to compliance. Archives of General Psychiatry, 36(8), 866-869.

Jamison, K., Gerner, R., Hammen, C., \& Padesky, C. (1980). Clouds and silver linings: Positive experiences associated with primary affective disorders. The American Journal of Psychiatry, 137(2), 198-202.

Jones, A. \& Sommerlund, B. (2007). A critical discussion of null hypothesis significance testing and statistical power analysis within psychological research. Nordic Psychology, 59(3), 223-230.

Judd, L.L. \& Akiskal, H.S. (2003). The prevalence and disability of bipolar spectrum disorders in the US population: Re-analysis of the ECA database taking into account subthreshold cases. Journal of Affective Disorders, 73, 123-131.

Keck, P., McElroy, S., Strakowski, S., \& Bourne, M. (1997). Compliance with maintenance treatment in bipolar disorder. Psychopharmacology Bulletin, 33(1), 87-91. 
Kinney, D., \& Richards, R. (2007). Artistic creativity and affective disorders: Are they connected? Evolutionary and neurocognitive approaches to aesthetics, creativity and the arts (pp. 225-237). Amityville, NY US: Baywood Publishing Co.

Lee, J. (2007). Melancholy, the muse and mental health promotion - An analysis of the complex relationship between mood disorder and creativity, developing a specific model of mental health promotion: Six key themes. International Journal of Mental Health Promotion, 9(1), 4-16.

Lingam, R., \& Scott, J. (2002). Treatment non-adherence in affective disorders. Acta Psychiatrica Scandinavica, 105(3), 164-172.

Makel, M.C. \& Plucker, J.A. (2008). Creativity. In S. Pfeiffer (Ed.), Handbook of Giftedness in Children: Psychoeducational Theory, Research, and Best Practices (p. 247-270). New York: Springer Press.

Mortenson, S.T. (2002). Sex, communication values, and cultural values: Individualismcollectivism as a mediator of sex differences in communication values in two cultures. Communication Reports, 15(1), 57-70.

Mumford, M.D. (2003). Where have we been, where are we going? Taking stock in creativity research. Creativity Research Journal, 15(2), 107-120.

Murphy, M. (2008). Pure art, pure desire: Changing definitions of l'art pour l'art from Kant to Gautier. Studies in Romanticism, 47(2), 147-160.

Noble, L. (1998). Doctor-patient communication and adherence to treatment. In L.B. Myers \& K. Midence (Eds.), Adherence to treatment in medical conditions (pp. 51-82). Amsterdam Netherlands: Harwood Academic Publishers. 
Nowakowska, C., Strong, C.M., Santosa, C.M., Wang, P.W., Ketter, T.A. (2005).

Temperamental commonalities and differences in euthymic mood disorder patients, creative controls, and healthy controls. Journal of Affective Disorders, 85(1), 207-215.

Pachet, A.K. (1999). Differential neuropsychological performances of bipolar mood disorder patients treated with divalproex sodium or lithium carbonate. Dissertation Abstracts International, 60, Retrieved from PsychINFO database.

Park, H.S., Levine, T.R., Westerman, C.Y.K., Orfgen, T., \& Foregger, S. (2007). The effects of argument quality and involvement type on attitude formation and attitude change: A test of dual-process and social judgment predictions. Human Communication Research, 33, 81-102.

Plucker, J., Beghetto, R., \& Dow, G. (2004). Why isn't creativity more important to educational psychologists? Potentials, pitfalls, and future directions in creativity research. Educational Psychologist, 39(2), 83-96.

Prentky, R.A. (2001). Mental illness and roots of genius. Creativity Research Journal, 13(1), 95104.

Qian, M., Plucker, J.A., \& Shen, J. (2010). A model of Chinese adolescents' creative personality. Creativity Research Journal, 22(1), 62-67.

Richards, R. (2001). Creativity and the schizophrenia spectrum: More and more interesting. Creativity Research Journal, 13(1), 111-132.

Richards, R. \& Kinney, D. (1990). Mood swings and creativity. Creativity Research Journal, 3(3), 202-217. 
Richards, R., Kinney, D., Daniels, H., \& Linkins, K. (1992). Everyday creativity and bipolar and unipolar affective disorder: Preliminary study of personal and family history. European Psychiatry, 7(2), 49-52.

Richards, R., Kinney, D., Lunde, I., Benet, M., \& Merzel, A. (1988). Creativity in manicdepressives, cyclothymes, their normal relatives, and control subjects. Journal of Abnormal Psychology, 97(3), 281-288.

Rothenberg, A. (2001). Bipolar illness, creativity, and treatment. Psychiatric Quarterly (New York), 72(2), 131-147.

Ryan, R.M., La Guardia, J.G., Solky-Butzel, J., Chirkov, V., \& Kim, Y. (2005). On the interpersonal regulation of emotions: Emotional reliance across gender, relationships, and cultures. Personal Relationships, 12, 145-163.

Santosa, C., Strong, C., Nowakowska, C., Wang, P., Rennicke, C., \& Ketter, T. (2007). Enhanced creativity in bipolar disorder patients: A controlled study. Journal of Affective Disorders, 100(1), 31-39.

Sasaki, J.Y. \& Kim, H.S. (2011). At the intersection of culture and religion: A cultural analysis of religion's implications for secondary control and social affiliation. Journal of personality and social psychology, 101(2), 401-414.

Schou, M. (1979). Artistic productivity and lithium prophylaxis in manic-depressive illness. British Journal of Psychiatry, 135, 97-103.

Schuldberg, D. (1990). Schizotypal and hypomanic traits, creativity, and psychological health. Creativity Research Journal, 3(3), 218-230.

Schuldberg, D. (2001). Six subclinical spectrum traits in normal creativity. Creativity Research Journal, 13(1), 5-16. 
Schwab, M. (2008). First, the second: Walter benjamin's theory of reflection and the question of artistic research. Journal of Visual Arts Practice, 7(3), 213-223.

Scott, J. (2002). Using health belief models to understand the efficacy-effectiveness gap for mood stabilizer treatments. Neuropsychobiology, 46, 13-15.

Scott, J., \& Pope, M. (2001). Nonadherence with mood stabilizers: Prevalence and predictors. Journal of Clinical Psychiatry, 63(5), 384-390.

Shaw, E., Mann, J., Stokes, P., \& Manevitz, A. (1986). Effects of lithium carbonate on associative productivity and idiosyncrasy in bipolar outpatients. American Journal of Psychiatry, 143(9), 1166-1169.

Shaw, E., Stokes, P., Mann, J., \& Manevitz, A. (1987). Effects of lithium carbonate on the memory and motor speed of bipolar outpatients. Journal of Abnormal Psychology, 96(1), 64-69.

Shulruf, B., Alesi, M., Ciochina, L., Faria, L., Hattie, J., Hong, F., Pepi, A., \& Watkins, D. (2011). Measuring collectivism and individualism in the third millennium. Social behavior and personality, 39(2), 173-188.

Simonton, D.K. (1999). Origins of genius: Darwinian perspectives on creativity. New York, NY US: Oxford University Press.

Simonton, D.K. (2002). Creativity. In C.R. Snyder \& S.J. Lopez (Eds.), Handbook of positive psychology (pp. 189-201). New York: Oxford University Press.

Smith, D.J., Ghaemi, S.N., \& Craddock, N. (2008). The broad clinical spectrum of bipolar disorder: Implications for research and practice. Journal of Psychopharmacology, 22(4), $397-400$. 
Smith, G.J. (2005). How should creativity be defined? Creativity Research Journal, 17(2), 293295.

Srivastava, S., Childers, M., Baek, J.H., Strong, C.M., Hill, S.J., Warsett, K.S., Wang, P.W., Akiskal, H.S., Akiskal, K.K., \& Ketter, T.A. (2010). Toward interaction of affective and cognitive contributors to creativity in bipolar disorders: A controlled study. Journal of Affective Disorders (2010), doi: 10.1016/j.jad.2009.12.018

Stahl, S.M. (2008). Stahl's essential psychopharmacology: Neuroscientific basis and practical applications. New York: Cambridge University Press.

Sternberg, R.J. (2006). Introduction. In J.C. Kaufman \& R.J. Sternberg (Eds.), The International Handbook of Creativity (pp. 1-10). New York: Cambridge University Press.

Stevens, J. P. (2009). Applied multivariate statistics for the social sciences (5th ed.). Mahwah, NJ: Routledge Academic.

Strong, C.M., Nowakowska, C., Santosa, C.M., Wang, P.W., Kraemer, H.C., Ketter, T.A. (2007). Temperament-creativity relationships in mood disorder patients, healthy controls and highly creative individuals. Journal of Affective Disorders, 100(1), 41-48.

Sun, S., Pan, W., \& Wang, L.L. (2011). Rethinking observed power: Concept, practice, and implications. Methodology, 7(3), 81-87.

Svrakic, D.M., Draganic, S., Hill, K., Bayon, C., Przybeck, T.R., Cloninger, C.R. (2002). Temperament, character, and personality disorders: Etiologic, diagnostic, treatment issues. Acata Psychiatrica Scandinavica, 106, 189-195.

Svrakic, N.M., Svrakic, D.M., \& Cloninger, C.R. (1996). A general quantitative theory of personality development: Fundamentals of a self-organizing psychobiological complex. Development and Psychopathology, 8, 247-272. 
Thompson, C., Peveler, R.C., Stephenson, D., \& McKendrick (2000). Compliance with antidepressant medications in the treatment of major depressive disorder in primary care: A randomized comparison of fluoxetine and a tricyclic antidepressant. American Journal of Psychiatry, 15(7), 338-343.

Waddell, C. (1998). Creativity and mental illness: Is there a link? Canadian Journal of Psychiatry, 43, 166-172.

Widiger, T.A. \& Mullins-Sweatt, S. (2007). Mental disorders as discrete clinical conditions: dimensional versus categorical classification. In M. Hersen, S.M. Turner, D.C. Beidel (Eds), Adult Psychopathology and Diagnosis (fifth edition) (pp. 3-34). Hoboken, N.J.: John Wiley \& Sons, Inc. 
Appendices 
Appendix A: Descriptors for high and low scores on the four temperament dimensions of the TCI-R (Cloninger, 2004) with associated Cronbach Alpha coefficients (Gonclaves \& Cloninger, 2010).

\begin{tabular}{|l|l|l|l|}
\hline Temperament & High Score & Low Score & Cronbach Alpha \\
\hline Dimension & & & \\
\hline Harm Avoidance & Anxious & Risk Taking & .84 \\
\hline subscale 1 (HA1) & Pessimistic & Optimistic & .65 \\
\hline HA3 & Fearful & Daring & \\
\hline HA4 & Shy & Outgoing & .59 \\
\hline Novelty Seeking & Fatigable & Vigorous & .72 \\
\hline Novelty Seeking & Exploratory & Stoical & .71 \\
\hline subscale 1 (NS1) & & Reserved & .71 \\
\hline NS2 & Impulsive & & .48 \\
\hline NS3 & Extravagant & Frugal & .68 \\
\hline NS4 & Irritable & Stoic & .59 \\
\hline Reward Dependence & Gregarious & Stoical & .74 \\
\hline Reward Dependence & Sentimental & Critical & .69 \\
\hline subscale 1 (RD1) & & & .47 \\
\hline RD2 & Sociable & Aloof & \\
\hline RD3 & Sympathetic & Detached & \\
\hline
\end{tabular}




\begin{tabular}{|l|l|l|l|}
\hline Persistence & Industriousness & Underachieving & .86 \\
\hline Persistence subscale 1 & Eager & Apathetic & .56 \\
\hline PS1) & & & \\
\hline PS2 & Determined & Spoiled & .65 \\
\hline PS4 & Ambitious & Underachiever & .71 \\
\hline
\end{tabular}

Descriptors for high and low scores on the three character dimensions of the TCI-R

(Cloninger, 2004) with associated Cronbach Alpha coefficients (Gonclaves \& Cloninger,

2010).

\begin{tabular}{|l|l|l|l|}
\hline Character Dimension & High Score & Low Score & Cronbach Alpha \\
\hline Self-Directedness & Responsible & Externalizing & .87 \\
\hline subscale 1 (SD1) & Responsible & Blaming & .71 \\
\hline SD2 & Purposeful & Aimless & \\
\hline SD3 & Resourceful & Inept & .63 \\
\hline SD4 & Self-Accepting & Vain & .64 \\
\hline SD5 & Hopeful & Deliberating & .72 \\
\hline Cooperativeness & Empathic & Selfish & .69 \\
\hline Cooperative subscale 1 & Reasonable & Prejudiced & .63 \\
(C1) & & & .69 \\
\hline C2 & Empathic & Insensitive & .45 \\
\hline C3 & Helpful & Hostile & .48 \\
\hline
\end{tabular}




\begin{tabular}{|l|l|l|l|}
\hline C4 & Compassionate & Revengeful & .80 \\
\hline C5 & Principled & Opportunistic & .49 \\
\hline Self-Transcendence & Humble/Fulfilled & Proud/Unfulfilled & .84 \\
\hline Self-Transcendent & Judicious & Repressive & .68 \\
\hline ST1 $)$ & & & \\
\hline ST2 & Idealistic & Practical & .73 \\
\hline ST3 & Transpersonal & Dualistic & .72 \\
\hline ST4 & Faithful & Skeptical & Not Provided \\
\hline ST5 & Spiritual & Materialistic & Not Provided \\
\hline
\end{tabular}


Appendix B: Types of Reliability and Validity for BWAS and TCI-R

\begin{tabular}{|l|l|}
\hline BWAS & \\
\hline Reliability & Validity \\
\hline Split-Half & Criterion (known-groups) \\
\hline Test-Retest (temporal stability) & \\
\hline
\end{tabular}

\begin{tabular}{|l|l|}
\hline TCI-R & \\
\hline Reliability & Validity \\
\hline Internal Consistency & Criterion (concurrent) \\
\hline Principle Component Analysis of & Convergent \\
Temperament and Character dimensions & \\
\hline
\end{tabular}


Appendix C: Sample from BWAS (sample reprinted with permission)

For use by Clark Pinson only. Received from Mind Garden, Inc. on January 27, 2011

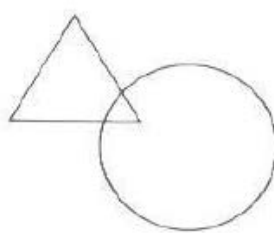

1

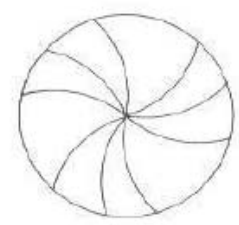

2

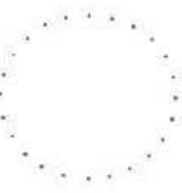

3

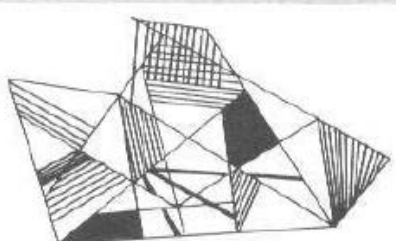

5

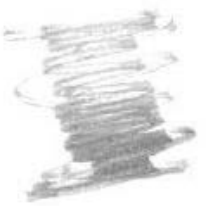

6

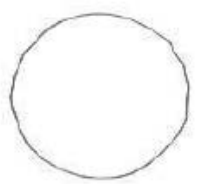

7 


\section{Appendix D: Sample from TCI-R (sample reprinted with permission)}

Copyright (C) 1987, 1992, 1996, 1999 C. R. Cloninger. All rights reserved.

1

\section{TCI-R}

For each of the following questions, please circle the number that best describes the way you usually or generally act or feel. (Circle only one number for each question)

\begin{tabular}{|c|c|c|c|c|}
\hline $\mathbf{1}$ & $\mathbf{2}$ & $\mathbf{3}$ & $\mathbf{4}$ & $\mathbf{5}$ \\
$\begin{array}{c}\text { Definitely } \\
\text { False }\end{array}$ & $\begin{array}{c}\text { Mostly or } \\
\text { Probably False }\end{array}$ & $\begin{array}{c}\text { Neither true nor False, } \\
\text { or about equally } \\
\text { True or False }\end{array}$ & $\begin{array}{c}\text { Mostly or } \\
\text { Probably True }\end{array}$ & $\begin{array}{c}\text { Definitely } \\
\text { True }\end{array}$ \\
\hline
\end{tabular}

\begin{tabular}{|c|c|c|c|c|c|c|}
\hline 1 & $\begin{array}{l}\text { I often try new things just for fun or thrills, even if most people think it is a waste } \\
\text { of time. }\end{array}$ & 1 & 2 & 3 & 4 & 5 \\
\hline 2 & $\begin{array}{l}\text { I usually am confident that everything will go well, even in situations that worry } \\
\text { most people. }\end{array}$ & 1 & 2 & 3 & 4 & 5 \\
\hline 3 & I often feel that I am the victim of circumstances. & 1 & 2 & 3 & 4 & 5 \\
\hline 4 & $\begin{array}{l}\text { I can usually accept other people as they are, even when they are very different } \\
\text { from me. }\end{array}$ & 1 & 2 & 3 & 4 & 5 \\
\hline 5 & I like a challenge better than easy jobs. & 1 & 2 & 3 & 4 & 5 \\
\hline 6 & Often I feel that my life has little purpose or meaning. & 1 & 2 & 3 & 4 & 5 \\
\hline 7 & I like to help find a solution to problems so that everyone comes out ahead. & 1 & 2 & 3 & 4 & 5 \\
\hline 8 & I am usually eager to get going on any job I have to do. & 1 & 2 & 3 & 4 & 5 \\
\hline 9 & $\begin{array}{l}\text { I often feel tense and worried in unfamiliar situations, even when others feel } \\
\text { there is little to worry about. }\end{array}$ & 1 & 2 & 3 & 4 & 5 \\
\hline 10 & $\begin{array}{l}\text { I often do things based on how I feel at the moment without thinking about how } \\
\text { they were done in the past. }\end{array}$ & 1 & 2 & 3 & 4 & \begin{tabular}{|l|}
5 \\
\end{tabular} \\
\hline
\end{tabular}




\title{
Appendix E: Demographic Questionnaire
}

\author{
Demographic Questionnaire
}

1. What is your gender?

Male

Female

2. What is your age (you must be 18 years or older)?

3. What is your current relationship status (please check only one answer)?

Single (never married)

In a committed relationship

Married

Widowed (not currently married)

Divorced (not remarried)

Other (please specify)

4. What is your race (please check only one)?

American Indian/Alaska Native

Asian

Black/African American

Latino/Hispanic/Chicano

Middle Eastern

Native Hawaiian/Other Pacific Islander

White

Multirracial (please specify)

Other (please specify)

5. What is the highest level of education that you have completed (please check only one answer)?

High School

College B.A. or B.S.

College M.A., M.S., or M.S.W.

College Ph.D., Psy.D., Ed.D., J.D., or M.D.

Other (please specify)

6. What is (or was) your major? 


\title{
Appendix F: Cover Letter
}

\author{
WestVirginiaUniversity \\ College of Human Resources and Education
}

Dear Participant,

This letter is a request for you to take part in a research project to assess how aspects of your temperament relate to certain facets of creativity. Temperament represents what a person focuses his or her attention on and how intensely he/she responds to the subject of focus. This project is being conducted by Clark Pinson, MA and represents his doctoral dissertation in Counseling Psychology. He is being supervised by Dr. Jeff Daniels, Ph.D., a professor in the WVU department of Counseling, Rehabilitation Counseling and Counseling Psychology. Your participation in this project is greatly appreciated and will take approximately 1 hour to complete the three attached questionnaires.

Your involvement in this project will be kept as confidential as legally possible. All data will be reported in the aggregate. You must be 18 years of age or older to participate. I will not ask any information that should lead back to your identity as a participant. Your participation is completely voluntary. You may skip any question that you do not wish to answer and you may discontinue at any time. Your class standing will not be affected if you decide either not to participate or to withdraw. West Virginia University's Institutional Review Board acknowledgement of this project is on file.

If you have any questions about this letter or the research project, please feel free to contact Jeff Daniels, Ph.D. at (304) 293-2235 or by e-mail at Jeffrey.Daniels@mail.wvu.edu. You can also contact Clark Pinson, MA at (304) 293-4431 or by email at cpinson1@mix.wvu.edu.

Thank you for your time and help with this project.

Sincerely,

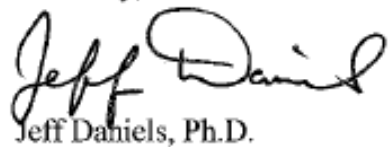

Yeff Dahiels, $\mathrm{Ph} . \mathrm{D}$.

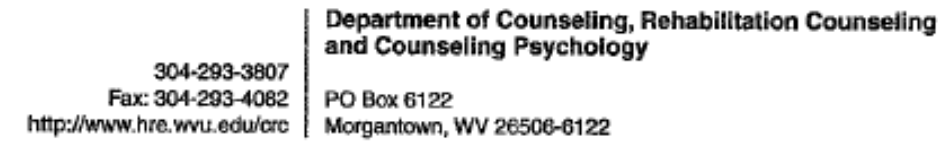

Equal Opportunity/Atfirmative Action Institution 